\title{
Strong decays of higher charmonium states into open-charm meson pairs
}

\author{
Long-Cheng Gui, ${ }^{1,2,{ }^{*}}$ Long-Sheng Lu, ${ }^{1}$ Qi-Fang Lü, ${ }^{1,2, \dagger}$ Xian-Hui Zhong, ${ }^{1,2, \$}$ and Qiang Zhao ${ }^{3,2,4,8}$ \\ ${ }^{1}$ Department of Physics, Hunan Normal University, and Key Laboratory of Low-Dimensional Quantum \\ Structures and Quantum Control of Ministry of Education, Changsha 410081, China \\ ${ }^{2}$ Synergetic Innovation Center for Quantum Effects and Applications (SICQEA), \\ Hunan Normal University, Changsha 410081, China \\ ${ }^{3}$ Institute of High Energy Physics and Theoretical Physics Center for Science Facilities, \\ Chinese Academy of Sciences, Beijing 100049, China \\ ${ }^{4}$ School of Physical Sciences, University of Chinese Academy of Sciences, Beijing 100049, China
}

(Received 1 February 2018; published 18 July 2018)

\begin{abstract}
The open-charm strong decays of higher charmonium states up to the mass of the $6 P$ multiplet are systematically studied in the ${ }^{3} P_{0}$ model. The wave functions of the initial charmonium states are calculated in the linear potential and screened potential quark models. The decay widths for most of the wellestablished charmonium states above the open-charm thresholds can be reasonably described. By comparing our quark model calculations with the experimental observations we also discuss the nature of some of the newly observed charmonium-like states. It is found that (i) the $\psi(4415)$ may favor the $\psi(4 S)$ or $\psi_{1}(3 D)$ assignment. There may exist two highly overlapping vector charmonium states around $4.4 \mathrm{GeV}$; (ii) in the LP model the $J^{P C}=1^{--} Y(4660)$ resonance and the $J^{P C}=0^{++} X(4500)$ resonance may be assigned as the $\psi(5 S)$ and $\chi_{c 0}(4 P)$, respectively; (iii) the newly observed state $X^{*}(3860)$ can be assigned as the $\chi_{c 0}(2 P)$ state with a narrow width of about $30 \mathrm{MeV}$; (iv) it seems to be difficult to accommodate the $X(4140)$ and $X(4274)$ states in the same potential model as excited $\chi_{c 1}$ states; (v) the $X(3940)$ resonance can be assigned as the $\eta_{c}(3 S)$ state; (vi) the vector charmonium-like states $Y(4230 / 4260,4360)$ and scalar $X(4700)$ cannot be described by any conventional charmonium states self-consistently in our model.
\end{abstract}

DOI: $10.1103 /$ PhysRevD.98.016010

\section{INTRODUCTION}

During the past decade, many new charmonium-like states above/near open-charm thresholds, such as $X(3940)$, $X(4140 / 4274), \quad Y(4230 / 4260), \quad Y(4360), \quad Y(4660)$, $X(4500)$ and $X(4700)$, have been reported by the Belle, $B A B A R$, LHCb, BESIII, CLEO collaborations and so on [1]. Lately, a new charmonium-like state $X^{*}(3860)$ was observed in the $e^{+} e^{-} \rightarrow J / \psi D \bar{D}$ process by the Belle collaboration [2]. These newly observed charmonium-like states have attracted a lot of attention from the hadron physics community. One obvious feature is that most of them have masses located around $S$-wave open-flavor thresholds and cannot be easily accommodated by the conventional quark model. Because of this, they have

\footnotetext{
*guilongcheng@hunnu.edu.cn

†vqifang@hunnu.edu.cn

zhongxh@hunnu.edu.cn

${ }^{\S}$ zhaoq@ihep.ac.cn
}

Published by the American Physical Society under the terms of the Creative Commons Attribution 4.0 International license. Further distribution of this work must maintain attribution to the author(s) and the published article's title, journal citation, and DOI. Funded by SCOAP. initiated tremendous interests and different ideas. Detailed reviews on the status of these charmonium-like states can be found in Refs. [3-9], where some of these states are categorized as exotic hadrons.

Although there has been progress made during the past years, there still exist many mysteries to be uncovered. As we have known that exotic states with normal quantum numbers (e.g., tetraquark states [3], hadronic molecules [6], charmonium hybrids [10], and so on) can hardly be distinguished from the normal ones, in order to understand exotic candidates, one should also have a reasonable description of the normal hadron spectrum. In the charmonium sector the low-lying states can be very well described by nonrelativistic potential quark model such as the Cornell model [11] and Godfrey-Isgur model [12-14]. However, it is realized that the open channel effects would become essential for higher excited states and it is still challenging to include such effects in a coherent way $[13,15]$.

In order to understand these recently observed charmonium-like states, a better understanding of the charmonium spectrum can be regarded as a prerequisite. In this work we take the strategy of studying systematically the charmonium open flavor decays within the widely used linear potential (LP) model [12-14] and screened potential (SP) 
model $[15,16]$ such that most of the conventional charmonium states can be identified by comparing with the experimental measurements. Although it should be recognized that the SP model may not be sufficient for including the full open threshold effects, we anticipate that unusual phenomena arising from such a study would indicate signals for unconventional structures of some of those charmonium-like states. In Ref. [17] the charmonium spectrum and their electromagnetic (EM) transitions have been studied within both LP and SP models. For the lowlying charmonium states with a mass of $M<4.0 \mathrm{GeV}$, both models give comparable predictions. However, for the higher charmonium states with a mass of $M>4.1 \mathrm{GeV}$, the SP model gives very different results from the LP model. For example, in the SP model, the $J^{P C}=1^{--}$ charmonium-like states $Y(4260)$ and $Y(4360)$ are good candidates for the $\psi(4 S)$ and $\psi_{1}(3 D)$ states, respectively, while the $J^{P C}=1^{++}$charmonium-like states $X(4140)$ may be assigned as the $\chi_{c 1}(3 P)$ state. In contrast, there is no room for the charmonium-like states $Y(4260), Y(4360)$ and $X(4140)$ in the LP model, the well-established state $\psi(4415)$ may be assigned to $\psi(4 S)$ or $\psi_{1}(3 D)$, and the charmonium-like states $X(4274)$ seem to be a candidate of $\chi_{c 1}(3 P)$. Such a result has already shown different dynamic origins introduced by the color screening effects. To clarify the nature of the newly observed charmonium-like states, we continue to investigate the open-flavor strong decays in the SP and LP models in this work. The differences between these two models and their comparisons with experimental observations can provide valuable information on the internal structures of these charmonium-like states.

By adopting the wave functions of the charmonium states calculated with the LP and SP models in our previous work [17] their strong decay amplitudes can be calculated by the widely used ${ }^{3} P_{0}$ model [18-24]. In this method, one assumes that a $q \bar{q}$ pair is produced from the vacuum with the vacuum quantum numbers, $J^{P C}=0^{++}$, and the decay of the charmonium state takes place by regrouping the new $q \bar{q}$ pair created from the vacuum and the $c \bar{c}$ in the initial state into the outgoing open-charm meson pair via a rearrangement process.

As an important topic in hadron physics, the open-charm strong decays of the charmonium states are often discussed in the literature [13,22-46]. Several pioneering works can be found in Refs. [22-24], where the open charm strong decays of $\psi(3770), \psi(4040), \psi(4160)$ and $\psi(4415)$ have been evaluated about forty years ago. Stimulated by first observed charmonium-like resonance $X(3872)$ at Belle [47] and CDF [48], Eichten, Lane and Quigg analyzed the open charm strong decays of charmonium states near threshold in the Cornell coupled-channel model [36,37]. In 2007, Ding, Zhu and Yan considered the open flavor strong decays of $Y(4360)$ and $Y(4660)$ as $3^{3} D_{1}$ and $5^{3} S_{1}$ canonical charmonium in the simple harmonic oscillator wave function approximation in the framework of flux tube model [38]. In 2008, Segovia et al. calculated the openflavor strong decays of the $J^{P C}=1^{--}$charmonium states in the ${ }^{3} P_{0}$ model [25], where the new $X(4360)$ state was considered to be the $\psi(4 S)$ state and the $\psi(4415)$ as the $\psi_{1}(3 D)$ state, which differs from other assignments. In 2009, as conventional charmonium states, the open-flavor strong decays of the newly observed resonances $X(3915)$ and $X(4350)$ were studied by Liu et al. within the ${ }^{3} P_{0}$ model, the strong decay properties indicate that they may be assigned as $\chi_{c 0}(2 P)$ and $\chi_{c 2}(3 P)$, respectively [44]. Further studies of the open-flavor strong decays of $P$-wave charmonium states were also carried out within the ${ }^{3} P_{0}$ model by several groups in recent years [27-30]. It is found that $X(3915)$ may be disfavored the assignment of $\chi_{c 0}(2 P)$ $[27,28], X(4140)$ may favor the $\chi_{c 1}(3 P)$ state [29], while the newly observed state $X^{*}(3860)$ can be a good candidate of $\chi_{c 0}(2 P)$ with a broad width [30]. Recently, the BetheSalpeter method was also extended to deal with the opencharm strong decays of several charmonium states [39-41]. However, as emphasized earlier, systematic studies of the full spectrum are essential for a better understanding of the underlying dynamics. Furthermore, how to properly treat the strong $S$-wave threshold interactions is a key issue for the description of near-threshold states [6]. The SP model can partially account for such an effect which makes the systematic comparison between the LP and SP model results interesting. Note that the most recent systematic study of the strong decays of higher charmonium states was carried out by Barnes, Godfrey and Swanson [13] quite long ago. It is necessary to reinvestigate in a systematic way the strong decays of the higher charmonium states by combining the recent progress in theory and experiments.

The paper is organized as follows. In Sec. II, a brief introduction to the ${ }^{3} P_{0}$ strong decay model is presented. In Sec. III, we focus on the calculation results and discuss the phenomenological consequences in comparison with the experimental data. A summary is given in Sec. IV.

\section{II. ${ }^{3} \boldsymbol{P}_{\mathbf{0}}$ MODEL}

In this work, we used the ${ }^{3} P_{0}$ model to calculate the Okubo-Zweig-Iizuka (OZI) allowed strong decay widths for the charmonium states above $D \bar{D}$ threshold. The ${ }^{3} P_{0}$ model is a model that describes the quark pair creation mechanism of the OZI allowed strong decays based on the quark model. It is firstly proposed by Micu [18] and then extended by Le Yaouanc et al. [19,20]. This model has been widely applied to deal with the open-charm strong decays of the charmonium states $[13,22-34,46]$. In the ${ }^{3} P_{0}$ model, one assumes that a quark-antiquark pair is produced from the vacuum with the quantum number $0^{++}$and the heavy meson decay takes place via the rearrangement of the four quarks. Such a process is empirically illustrated in Fig. 1. 


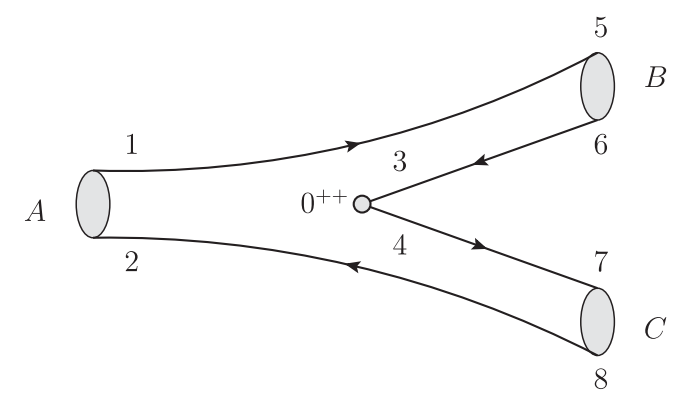

FIG. 1. The strong decay mechanism for meson two-body decays $A \rightarrow B C$ in the ${ }^{3} P_{0}$ model.

The quark pair creation process from vacuum can be described as

$$
\begin{aligned}
T= & -3 \gamma \sqrt{96 \pi} \sum_{m}\langle 1 m 1-m \mid 00\rangle \int d \mathbf{p}_{3} d \mathbf{p}_{4} \delta^{3}\left(\mathbf{p}_{3}+\mathbf{p}_{4}\right) \\
& \times \mathcal{Y}_{1}^{m}\left(\frac{\mathbf{p}_{3}-\mathbf{p}_{4}}{2}\right) \chi_{1-m}^{34} \phi_{0}^{34} \omega_{0}^{34} b_{3 i}^{\dagger}\left(\mathbf{p}_{3}\right) d_{4 j}^{\dagger}\left(\mathbf{p}_{4}\right),
\end{aligned}
$$

where $\gamma$ is a dimensionless constant that denotes the strength of the quark-antiquark pair creation with momentum $\mathbf{p}_{3}$ and $\mathbf{p}_{4}$ from vacuum; $b_{3 i}^{\dagger}\left(\mathbf{p}_{3}\right)$ and $d_{4 j}^{\dagger}\left(\mathbf{p}_{4}\right)$ are the creation operators for the quark and antiquark, respectively; the subscriptions, $i$ and $j$, are the $\mathrm{SU}(3)$-color indices of the created quark and antiquark; $\phi_{0}^{34}=(u \bar{u}+d \bar{d}+s \bar{s}) / \sqrt{3}$ and $\omega_{0}^{34}=\frac{1}{\sqrt{3}} \delta_{i j}$ correspond to flavor and color singlets, respectively; $\chi_{1,-m}^{34}$ is a spin triplet state; and $\mathcal{Y}_{\ell m}(\mathbf{k}) \equiv$ $|\mathbf{k}|^{\ell} Y_{\ell m}\left(\theta_{\mathbf{k}}, \phi_{\mathbf{k}}\right)$ is the $\ell$ th solid harmonic polynomial. The factor $(-3)$ is introduced for convenience, which will cancel the color factor.

In the center-of-mass (c.m.) frame of the initial meson $A$, the helicity amplitude can be written as

$$
\begin{aligned}
& \mathcal{M}^{M_{J_{A}} M_{J_{B}} M_{J_{C}}}(\mathbf{P}) \\
& =\gamma \sqrt{96 \pi} \sum_{\substack{M_{L_{A}, M_{S_{A}}} \\
M_{L_{B}}, S_{S_{B}}, M_{L_{C}, M_{S_{C}}, m}}} I_{M_{L_{B}}, M_{L_{C}}}^{M_{L_{1}}, m}(\mathbf{P})\left\langle L_{A} M_{L_{A}} ; S_{A} M_{S_{A}} \mid J_{A} M_{J_{A}}\right\rangle \\
& \times\langle 1 m ; 1-m \mid 00\rangle\left\langle L_{B} M_{L_{B}} ; S_{B} M_{S_{B}} \mid J_{B} M_{J_{B}}\right\rangle \\
& \times\left\langle L_{C} M_{L_{C}} ; S_{C} M_{S_{C}} \mid J_{C} M_{J_{C}}\right\rangle\left\langle\phi_{B}^{13} \phi_{C}^{24} \mid \phi_{A}^{12} \phi_{0}^{34}\right\rangle \\
& \times\left\langle\chi_{S_{B} M_{S_{B}}^{13}}^{13} \chi_{S_{C} M_{S_{C}}}^{24} \mid \chi_{S_{A} M_{S_{A}}^{12}} \chi_{1-m}^{34}\right\rangle,
\end{aligned}
$$

with the integral in the momentum space,

$$
\begin{aligned}
I_{M_{L_{B}}, M_{L_{C}}}^{M_{L_{A}}, m}(\mathbf{P})= & \int d^{3} \mathbf{p}_{3} \Psi_{n_{B} L_{B} M_{L_{B}}}^{*}\left(\frac{m_{3} \mathbf{P}}{m_{1}+m_{3}}-\mathbf{p}_{3}\right) \\
& \times \Psi_{n_{C} L_{C} M_{L_{C}}}^{*}\left(\frac{-m_{3} \mathbf{P}}{m_{2}+m_{3}}+\mathbf{p}_{3}\right) \Psi_{n_{A} L_{A} M_{L_{A}}} \\
& \times\left(\mathbf{P}-\mathbf{p}_{3}\right) \mathcal{Y}_{1 m}\left(\mathbf{p}_{3}\right) .
\end{aligned}
$$

In the above equations, $\left(J_{A}, J_{B}\right.$ and $\left.J_{C}\right),\left(L_{A}, L_{B}\right.$ and $\left.L_{C}\right)$ and $\left(S_{A}, S_{B}\right.$ and $\left.S_{C}\right)$ are the quantum numbers of the total angular momenta, orbital angular momenta and total spin for hadrons $A, B, C$, respectively; in the c.m. frame of hadron $A$, the momenta $\mathbf{P}_{B}$ and $\mathbf{P}_{C}$ of mesons $B$ and $C$ satisfy $\mathbf{P}_{B}=-\mathbf{P}_{C} \equiv \mathbf{P} ; m_{1}$ and $m_{2}$ are the constituent quark masses of the initial hadron $A ; m_{3}$ is the mass of the antiquark created from vacuum; $\Psi_{n_{A} L_{A} M_{L_{A}}}, \Psi_{n_{B} L_{B} M_{L_{B}}}$ and $\Psi_{n_{C} L_{C} M_{L_{C}}}$ are the radial wave functions of hadrons $A, B$ and $C$, respectively, in the momentum space, while $\phi_{A}^{12}, \phi_{B}^{13}$ and $\phi_{C}^{24}\left(\chi_{S_{A} M_{S_{A}}}^{12}, \chi_{S_{B} M_{S_{B}}}^{13}\right.$ and $\left.\chi_{S_{C} M_{S_{C}}}^{24}\right)$ are the flavor (spin) wave functions of hadrons $A, B$ and $C$, respectively; $\left\langle\phi_{B}^{13} \phi_{C}^{24} \mid \phi_{A}^{12} \phi_{00}^{34}\right\rangle$ and $\left\langle\chi_{S_{B} M_{S_{B}}}^{13} \chi_{S_{C} M_{S_{C}}}^{24} \mid \chi_{S_{A} M_{S_{A}}}^{12} \chi_{1-m}^{34}\right\rangle$ are the flavor and spin matrix elements, respectively; $\left\langle L_{A} M_{L_{A}}\right.$; $S_{A} M_{S_{A}}\left|J_{A} M_{J_{A}}\right\rangle$ and $\left\langle L_{B} M_{L_{B}} ; S_{B} M_{S_{B}} \mid J_{B} M_{J_{B}}\right\rangle,\left\langle L_{C} M_{L_{C}}\right.$; $S_{C} M_{S_{C}}\left|J_{C} M_{J_{C}}\right\rangle$ and $\langle 1 m ; 1-m \mid 00\rangle$ are the corresponding Clebsch-Gordan coefficients.

A partial wave amplitude can be obtained by using the Jacob-Wick formula [49]

$$
\begin{aligned}
\mathcal{M}^{J L}(A \rightarrow B C)= & \frac{\sqrt{4 \pi(2 L+1)}}{2 J_{A}+1} \sum_{M_{J_{B}}, M_{J_{C}}}\left\langle L 0 J M_{J_{A}} \mid J_{A} M_{J_{A}}\right\rangle \\
& \times\left\langle J_{B} M_{J_{B}} J_{C} M_{J_{C}} \mid J M_{J_{A}}\right\rangle \mathcal{M}^{M_{J_{A}} M_{J_{B}} M_{J_{C}}}(\mathbf{K}),
\end{aligned}
$$

where $M_{J_{A}}=M_{J_{B}}+M_{J_{C}}, \mathbf{J} \equiv \mathbf{J}_{B}+\mathbf{J}_{C}$ and $\mathbf{J}_{A} \equiv \mathbf{J}_{B}+\mathbf{J}_{C}+\mathbf{L}$.

Then the strong decay width for a given decay mode of meson $A$ is given by

$$
\Gamma=2 \pi|\mathbf{P}| \frac{E_{B} E_{C}}{M_{A}} \sum_{J L}\left|\mathcal{M}^{J L}\right|^{2},
$$

where $M_{A}$ is the mass of the initial hadron $A$, while $E_{B}$ and $E_{C}$ stand for the energies of final hadrons $B$ and $C$, respectively.

When calculating a decay width of a charmonium state, we adopt the numerical wave function for a charmonium state calculated by the LP and SP models from our previous work [17]. For the emitted charmed mesons in a decay process, such as $D$ and $D^{*}$, we use simple harmonic oscillator wave functions as an approximation,

$$
\begin{aligned}
\Psi_{n L M_{L}}(\mathbf{P})= & \frac{(-1)^{n}(-i)^{L}}{\beta^{(3 / 2)}} \sqrt{\frac{2 n !}{\Gamma(n+L+3 / 2)}}\left(\frac{P}{\beta}\right)^{L} \\
& \times e^{-\frac{P^{2}}{2 \beta^{2}} L_{n}^{L+1 / 2}}\left(\frac{P^{2}}{\beta^{2}}\right) Y_{L M_{L}}\left(\Omega_{P}\right),
\end{aligned}
$$

where $\beta$ is the universal harmonic oscillator parameter, and $L_{n}^{L+1 / 2}\left(\frac{p^{2}}{\beta^{2}}\right)$ is an associated Laguerre polynomial. 
To partly remedy the inadequacy of the nonrelativistic wave function as the momentum $\mathbf{P}$ increases, a commonly used Lorentz boost factor $\gamma_{f}$ is introduced into the decay amplitudes [50-53],

$$
\mathcal{M}(\mathbf{P}) \rightarrow \gamma_{f} \mathcal{M}\left(\gamma_{f} \mathbf{P}\right)
$$

where $\gamma_{f} \equiv M_{B} / E_{B}$. In most decays, the three momenta $\mathbf{P}$ carried by the final state mesons are relatively small, which means the nonrelativistic prescription is reasonable and corrections from the Lorentz boost are not drastic.

In our calculations, we set $m_{u}=m_{d}=330 \mathrm{MeV}, m_{s}=$ $450 \mathrm{MeV}$ and $m_{c}=1483 \mathrm{MeV}$ for the constituent quark masses. The masses of the well-established hadrons in the final states used in the calculations are adopted from the PDG [1]. In the present work, both $\beta$ and the pair creation strength $\gamma$ are considered as free parameters, which are determined by fitting the decay widths of the wellestablished charmonium states $\psi(3770), \psi(4040)$, $\psi(4160)$ and $\chi_{c 2}(2 P)$. If we adopt the wave function of

TABLE I. Open-charm strong decay widths (in $\mathrm{MeV}$ ) and decay amplitudes (in $\mathrm{GeV}^{-1 / 2}$ ) for the four established charmonium states $\psi(3770), \psi(4040), \psi(4160)$ and $\chi_{c 2}(3927)$. The experimental values are taken from the PDG [1].

\begin{tabular}{|c|c|c|c|c|c|c|}
\hline \multirow[b]{2}{*}{ State } & \multirow[b]{2}{*}{ Mode } & \multicolumn{2}{|c|}{ LP model } & \multicolumn{2}{|c|}{ SP model } & \multirow{2}{*}{$\begin{array}{c}\text { Amplitudes (LP/SP) } \\
{\left[\Gamma_{\exp }\right]}\end{array}$} \\
\hline & & $\Gamma_{\mathrm{th}}$ & $\mathrm{Br}(\%)$ & $\Gamma_{\mathrm{th}}$ & $\mathrm{Br}(\%)$ & \\
\hline \multirow[t]{3}{*}{$\psi(3770)$} & $D^{0} \bar{D}^{0}$ & 15 & 56 & 15 & 56 & \multirow{3}{*}{$\begin{array}{c}{ }^{1} P_{1}=0.0921 / 0.0917 \\
{ }^{1} P_{1}=0.086 / 0.0863 \\
{[27 \pm 1]}\end{array}$} \\
\hline & $D^{+} D^{-}$ & 12 & 44 & 12 & 44 & \\
\hline & Total & 27 & 100 & 27 & 100 & \\
\hline \multirow[t]{8}{*}{$\psi(4040)$} & $D^{0} \bar{D}^{0}$ & 1.2 & 2.0 & 2.5 & 4.4 & \multirow{8}{*}{$\begin{array}{c}{ }^{1} P_{1}=0.0156 / 0.0225 \\
{ }^{1} P_{1}=0.0139 / 0.0211 \\
{ }^{3} P_{1}=-0.0356 /-0.0186 \\
{ }^{3} P_{1}=-0.041 /-0.0244 \\
{ }^{1} P_{1}=-0.0277 /-0.0285 \\
{ }^{5} P_{1}=0.1236 / 0.1275 \\
{ }^{1} P_{1}=-0.0257 /-0.0268 \\
{ }^{5} P_{1}=0.1151 / 0.1199 \\
{ }^{1} P_{1}=0.0426 / 0.0377 \\
{[80 \pm 10]}\end{array}$} \\
\hline & $D^{+} D^{-}$ & 0.9 & 1.6 & 2.2 & 3.8 & \\
\hline & $D^{* 0} D^{0}$ & 4.7 & 7.9 & 1.3 & 2.3 & \\
\hline & $D^{*+} D^{-}$ & 6.0 & 10 & 2.1 & 38 & \\
\hline & $D^{* 0} D^{* 0}$ & 23 & 39 & 25 & 44 & \\
\hline & $D^{*+} D^{*-}$ & 18 & 30 & 19 & 34 & \\
\hline & $D_{s} D_{s}$ & 5.9 & 9.0 & 3.3 & 7.3 & \\
\hline & Total & 60 & 100 & 55 & 100 & \\
\hline \multirow[t]{12}{*}{$\psi(4160)$} & $D^{0} \bar{D}^{0}$ & 6.1 & 7.9 & 7.0 & 8.4 & \multirow{12}{*}{$\begin{array}{c}{ }^{1} P_{1}=0.0312 / 0.0333 \\
{ }^{1} P_{1}=0.0307 / 0.0332 \\
{ }^{3} P_{1}=-0.0166 /-0.0257 \\
{ }^{3} P_{1}=-0.015 /-0.0246 \\
{ }^{1} P_{1}=-0.0139 /-0.0061 \\
{ }^{5} P_{1}=0.0062 / 0.0027 \\
{ }^{5} F_{1}=0.0818 / 0.0848 \\
{ }^{1} P_{1}=-0.0154 /-0.0076 \\
{ }^{5} P_{1}=0.0069 / 0.0034 \\
{ }^{5} F_{1}=0.0803 / 0.0839 \\
{ }^{1} P_{1}=0.0056 /-0.007 \\
{ }^{3} P_{1}=-0.0548 /-0.0454 \\
\quad[70 \pm 10]\end{array}$} \\
\hline & $D^{+} D^{-}$ & 5.9 & 7.5 & 6.9 & 8.2 & \\
\hline & $D^{* 0} D^{0}$ & 1.4 & 1.9 & 3.5 & 4.1 & \\
\hline & $D^{*+} D^{-}$ & 1.2 & 1.5 & 3.1 & 3.8 & \\
\hline & $D^{0 *} D^{0 *}$ & 27 & 35 & 27 & 34 & \\
\hline & & & & & & \\
\hline & $D^{*+} D^{*-}$ & 26 & 34 & 28 & 33 & \\
\hline & & & & & & \\
\hline & & & & & & \\
\hline & & 0.6 & 0 . & & 0.3 & \\
\hline & $D_{s}{ }^{*} D_{s}$ & 10 & 13 & 2.7 & 7.8 & \\
\hline & Totol & 79 & 100 & 80 & 100 & \\
\hline \multirow[t]{5}{*}{$\chi_{c 2}(3927)$} & $D^{0} \bar{D}^{0}$ & 12 & 33 & 12 & 30 & \multirow{5}{*}{$\begin{array}{c}{ }^{1} D_{2}=-0.057 /-0.0566 \\
{ }^{1} D_{2}=-0.0573 /-0.0573 \\
{ }^{3} D_{2}=-0.0623 /-0.0688 \\
{ }^{3} D_{2}=-0.0556 /-0.0617 \\
{[24 \pm 6]}\end{array}$} \\
\hline & $D^{+} D^{-}$ & 12 & 32 & 12 & 30 & \\
\hline & $D^{* 0} D^{0}$ & 7.8 & 21 & 9.5 & 23 & \\
\hline & $D^{*+} D^{-}$ & 5.8 & 15 & 7.1 & 17 & \\
\hline & Total & 38 & 100 & 41 & 100 & \\
\hline
\end{tabular}

a charmonium state calculated using the LP model, we obtain $\beta=0.380 \mathrm{GeV}$ and $\gamma=0.234$. If we adopt the wave function of a charmonium state calculated using the SP model, we have $\beta=0.356 \mathrm{GeV}$ and $\gamma=0.217$, which are consistent with the LP results. With these parameters, the decay widths of $\psi(3770), \psi(4040), \psi(4160)$ and $\chi_{c 2}(2 P)$ can be reasonably described in both LP and SP models (see Table I). The strong decay properties for the higher charmonium states up to the mass of the $6 P$ multiplet have been listed in Tables II-IX.

\section{RESULTS AND DISCUSSION}

\section{A. Well-established $c \bar{c}$ states}

We choose four states above the $D \bar{D}$ threshold to determine the parameters in our model, i.e., $\psi(3770)$, $\psi(4040), \psi(4160)$, and $\chi_{c 2}(3927)$ which are broadly accepted as $1^{3} D_{1}, 3^{3} S_{1}, 2^{3} D_{1}$, and $2^{3} P_{2}$ states, respectively. The first three states, $\psi(3770), \psi(4040)$, and $\psi(4160)$, have been well established for a long time, while $\chi_{c 2}(3927)$ was observed in experiment quite recently. A good understanding of their strong decay properties is the starting point for our study of the strong decay properties of other charmonium states.

\section{1. $\psi(3770)$}

The $\psi(3770)$ is assigned to be the $1^{3} D_{1}$ charmonium state though a small $S$-wave component is allowed. This is the first $D$-wave vector charmonium state in the spectrum and located close to the $D \bar{D}$ threshold. In principle, the production of a $D$-wave state will be highly suppressed in $e^{+} e^{-}$annihilations due to the heavy quark spin symmetry (HQSS) constraint. However, as found by experiment, the production cross section for $\psi(3770)$ is actually sizable. It indicates quite large HQSS breakings in the charmonium sector mainly because the charm quark mass is not heavy enough. As a consequence, its non- $D \bar{D}$ branching ratio turns out to be much larger than a naive estimate based on the HQSS (see e.g., Refs. [56-58] for a modern view of this topical issue).

The dominant decay mode of $\psi(3770)$ into $D \bar{D}$ is driven by the $D$-wave component in its wave function. By treating it as a pure $1^{3} D_{1}$ state as the leading approximation, its strong decay properties can be well understood within both LP and SP models. Note that the differences of the momentum transfers between the charged and neutral $D \bar{D}$ meson pairs will introduce isospin breaking effects to the $\psi(3770) \rightarrow D \bar{D}$ couplings in the ${ }^{3} P_{0}$ model. Taking into account such isospin breaking effects, we obtain the partial width ratio between the two modes $D^{0} \bar{D}^{0}$ and $D^{+} D^{-}$,

$$
\frac{\Gamma\left(D^{0} \bar{D}^{0}\right)}{\Gamma\left(D^{+} D^{-}\right)} \simeq 1.25
$$


TABLE II. Open-charm strong decay properties for the $S$-wave charmonium states in the LP and SP models. The widths listed in the brackets are calculated with the masses of observations.

\begin{tabular}{|c|c|c|c|c|c|c|c|c|c|}
\hline \multirow[b]{2}{*}{$\underline{\text { State }}$} & \multirow[b]{2}{*}{ Decay mode } & \multicolumn{4}{|c|}{ LP model } & \multicolumn{2}{|c|}{ SP model } & \multirow{2}{*}{$\begin{array}{c}\text { Amplitudes }\left(\mathrm{GeV}^{-1 / 2}\right) \\
\mathrm{LP} / \mathrm{SP}\end{array}$} & \multirow{2}{*}{$\begin{array}{c}\text { Mass }(\mathrm{MeV}) \\
\mathrm{LP} / \mathrm{SP} \\
\end{array}$} \\
\hline & & $\Gamma_{\text {th }}(\mathrm{MeV})$ & $\mathrm{Br}(\%)$ & $\Gamma_{\text {th }}(\mathrm{MeV})[13]$ & $\operatorname{Br}(\%)[13]$ & $\Gamma_{\text {th }}(\mathrm{MeV})$ & $\mathrm{Br}(\%)$ & & \\
\hline \multirow[t]{3}{*}{$\eta_{c}(3 S)$} & $D^{*} D$ & 21 [79] & 28 & 47 & 59 & $5.7[40]$ & 100 & \multirow{3}{*}{$\begin{array}{l}{ }^{3} P_{0}=0.0749 / 0.0255 \\
{ }^{3} P_{0}=0.1838 / 0.0339\end{array}$} & \multirow{3}{*}{$\begin{array}{c}4048 / 4004 \\
{[3940 / 3940]^{\mathrm{a}}}\end{array}$} \\
\hline & $D^{*} D^{*}$ & $54[\cdots]$ & 72 & 33 & 41 & ...... & $\ldots$ & & \\
\hline & Total & 75 [79] & 100 & 80 & 100 & $5.7[40]$ & 100 & & \\
\hline \multirow[t]{12}{*}{$\psi(4 S)$} & $D D$ & 1.7 & 2.6 & 0.4 & 0.5 & 2.3 & 19 & \multirow{3}{*}{$\begin{array}{c}{ }^{1} P_{1}=0.0145 / 0.0181 \\
{ }^{3} P_{1}=0.008 / 0.0126 \\
{ }^{1} P_{1}=-0.0054 /-0.0074 \\
{ }^{5} P_{1}=0.0239 / 0.0332\end{array}$} & \multirow[t]{12}{*}{$4415 / 4281$} \\
\hline & $D^{*} D$ & 1.0 & 1.5 & 2.3 & 2.9 & 1.0 & 7.8 & & \\
\hline & $D^{*} D^{*}$ & 3.8 & 5.7 & 16 & 21 & 5.8 & 47 & & \\
\hline & $D_{s} D_{s}$ & 0.09 & 0.1 & 1.3 & 1.6 & 0.1 & 0.8 & ${ }^{1} P_{1}=0.0035 / 0.0042$ & \\
\hline & $D_{s}^{*} D_{s}$ & 3.3 & 5.0 & 2.6 & 3.3 & 3.1 & 26 & ${ }^{3} P_{1}=0.0239 / 0.0269$ & \\
\hline & $D_{s}^{*} D_{s}^{*}$ & 3.8 & 5.7 & 0.7 & 0.8 & 0.05 & 0.4 & ${ }^{1} P_{1}=0.0064 /-0.001$ & \\
\hline & & & & & & & & ${ }^{5} P_{1}=-0.0286 / 0.0047$ & \\
\hline & $D D_{1}$ & 12 & 18 & 31 & 40 & $\cdots$ & $\cdots$ & $\begin{array}{l}{ }^{3} S_{1}=0.00004 / \cdots \\
{ }^{3} D_{1}=0.0578 / \cdots\end{array}$ & \\
\hline & $D D_{1}^{\prime}$ & 16 & 24 & 1.0 & 1.2 & $\cdots$ & $\cdots$ & $\begin{array}{l}{ }^{3} S_{1}=-0.0678 / \cdots \\
{ }^{3} D_{1}=0.00004 / \cdots\end{array}$ & \\
\hline & $D D_{2}$ & 17 & 25 & 23 & 29 & $\cdots$ & $\ldots$ & ${ }^{5} D_{1}=-0.0746 / \cdots$ & \\
\hline & $D^{*} D_{0}$ & 8.7 & 13 & 0.0 & 0.0 & $\ldots$ & $\ldots$ & ${ }^{3} S_{1}=0.0531 / \cdots$ & \\
\hline & Total & 67 & 100 & 78 & 100 & 12 & 100 & & \\
\hline \multirow[t]{7}{*}{$\eta_{c}(4 S)$} & $D^{*} D$ & 0.3 & 0.5 & 6.3 & 10.3 & 0.5 & 2.8 & \multirow{7}{*}{$\begin{array}{c}{ }^{3} P_{0}=0.0067 /-0.0095 \\
{ }^{3} P_{0}=0.0303 / 0.0368 \\
{ }^{3} P_{0}=-0.0321 /-0.0332 \\
{ }^{3} P_{0}=-0.0199 / 0.0136 \\
{ }^{1} S_{0}=0.0731 / 0.0521 \\
{ }^{5} D_{0}=-0.1068 / \cdots\end{array}$} & \multirow[t]{7}{*}{$4388 / 4264$} \\
\hline & $D^{*} D^{*}$ & 5.6 & 8.5 & 14 & 22.9 & 6.5 & 34 & & \\
\hline & $D_{s}{ }^{*} D_{s}$ & 5.7 & 8.7 & 2.2 & 3.6 & 4.6 & 24 & & \\
\hline & $D_{s}^{*} D_{s}^{*}$ & 1.6 & 2.5 & 2.2 & 3.6 & 0.4 & 1.9 & & \\
\hline & $D D_{0}$ & 24 & 36.7 & 11 & 18.0 & 7.4 & 38 & & \\
\hline & $D D_{2}$ & 28 & 43.1 & 24 & 39.3 & $\cdots$ & $\cdots$ & & \\
\hline & Total & 66 & 100 & 61 & 100 & 19 & 100 & & \\
\hline \multirow[t]{19}{*}{$\psi(5 S)$} & $D D$ & $0.6[0.3]$ & 1.1 & $\cdots$ & $\cdots$ & $1.1[0.6]$ & 6.6 & ${ }^{1} P_{1}=0.0075 / 0.0113$ & \multirow{19}{*}{$\begin{array}{c}4711 / 4472 \\
{[4643 / 4415]^{\mathrm{a}}}\end{array}$} \\
\hline & $D^{*} D$ & $1.2[0.2]$ & 2.1 & $\cdots$ & $\cdots$ & $1.5[0.3]$ & 8.8 & ${ }^{3} P_{1}=0.0112 / 0.0137$ & \\
\hline & $D^{*} D^{*}$ & $0.0[0.7]$ & 0.0 & $\cdots$ & $\cdots$ & $0.2[1.4]$ & 0.9 & ${ }^{1} P_{1}=0.0001 /-0.001$ & \\
\hline & $D_{s} D_{s}$ & $0.0[0.04]$ & 0.0 & $\ldots$ & $\ldots$ & $0.0[0.04]$ & 0.0 & ${ }^{1} P_{1}=0.0002 /-0.0001$ & \\
\hline & $D_{s}^{*} D_{s}$ & $0.3[0.6]$ & 0.5 & $\ldots$ & $\ldots$ & $0.5[0.9]$ & 3.3 & ${ }^{3} P_{1}=0.055 / 0.0092$ & \\
\hline & $D_{s}^{*} D_{s}^{*}$ & $1.5[1.4]$ & 2.5 & $\cdots$ & $\cdots$ & $1.3[0.5]$ & 7.8 & ${ }^{1} P_{1}=0.003 / 0.0035$ & \\
\hline & & & & & & & & ${ }^{5} P_{1}=-0.0134 /-0.0155$ & \\
\hline & $D D_{1}$ & $0.06[0.6]$ & 0.1 & $\cdots$ & $\cdots$ & $1.0[1.6]$ & 6.1 & $\begin{array}{l}{ }^{3} S_{1}=0.00002 / 0.00002 \\
{ }^{3} D_{1}=-0.003 / 0.0152\end{array}$ & \\
\hline & $D D_{1}^{\prime}$ & $10[10]$ & 17.5 & $\ldots$ & $\ldots$ & $5.2[1.7]$ & 31 & ${ }^{3} S_{1}=-0.038 /-0.0349$ & \\
\hline & $D D_{2}$ & $0.12[1.6]$ & 0.2 & $\cdots$ & $\ldots$ & $1.7[1.0]$ & 9.9 & ${ }^{5} D_{1}=-0.0041 /-0.0206$ & \\
\hline & $D^{*} D_{0}$ & $11[10]$ & 18.3 & $\cdots$ & $\cdots$ & $3.8[0.5]$ & 23 & ${ }^{3} S_{1}=0.0392 / 0.0308$ & \\
\hline & $D^{*} D_{1}$ & $4.3[5.7]$ & 7.3 & $\cdots$ & $\cdots$ & $0.2[\cdots]$ & 1.4 & ${ }^{3} S_{1}=-0.00003 / 0.0$ & \\
\hline & & & & & & & & $\begin{array}{l}{ }^{3} D_{1}=0.0134 / 0.0052 \\
5 D_{1}=0.0232\end{array}$ & \\
\hline & $D^{*} D_{1}^{\prime}$ & $18[8.3]$ & 30.3 & $\ldots$ & $\ldots$ & $0.3[\cdots]$ & 1.5 & ${ }^{3} S_{1}=-0.0551 /-0.0113$ & \\
\hline & & & & & & & & ${ }^{5} D_{1}=-0.00002 / 0.0$ & \\
\hline & $D^{*} D_{2}$ & $12[7.8]$ & 20.1 & $\cdots$ & $\cdots$ & $0.0[\cdots]$ & 0.0 & ${ }^{3} D_{1}=0.0073 /-0.0001$ & \\
\hline & & & & & & & & ${ }^{5} D_{1}=-0.094 / 0.0002$ & \\
\hline & & & & & & & & ${ }^{7} D_{1}=-0.0445 / 0.0009$ & \\
\hline & Total & $58[47]$ & 100 & $\ldots$ & $\ldots$ & $17[8.4]$ & 100 & & \\
\hline
\end{tabular}


TABLE II. (Continued)

\begin{tabular}{|c|c|c|c|c|c|c|c|c|c|}
\hline \multirow[b]{2}{*}{ State } & \multirow[b]{2}{*}{ Decay mode } & \multicolumn{4}{|c|}{ LP model } & \multicolumn{2}{|c|}{ SP model } & \multirow{2}{*}{$\begin{array}{c}\text { Amplitudes }\left(\mathrm{GeV}^{-1 / 2}\right) \\
\text { LP/SP }\end{array}$} & \multirow{2}{*}{$\begin{array}{c}\text { Mass }(\mathrm{MeV}) \\
\text { LP/SP }\end{array}$} \\
\hline & & $\Gamma_{\text {th }}(\mathrm{MeV})$ & $\mathrm{Br}(\%)$ & $\Gamma_{\text {th }}(\mathrm{MeV})[13]$ & $\operatorname{Br}(\%)[13]$ & $\Gamma_{\text {th }}(\mathrm{MeV})$ & $\mathrm{Br}(\%)$ & & \\
\hline \multirow[t]{11}{*}{$\eta_{c}(5 S)$} & $D^{*} D$ & 0.5 & 0.7 & $\cdots$ & $\cdots$ & 1.7 & 11 & \multirow{11}{*}{$\begin{array}{c}{ }^{3} P_{0}=-0.0075 /-0.0147 \\
{ }^{3} P_{0}=0.0067 / 0.0065 \\
{ }^{3} P_{0}=-0.0099 /-0.0123 \\
{ }^{3} P_{0}=-0.0134 /-0.014 \\
{ }^{1} S_{0}=0.0404 / 0.0365 \\
{ }^{5} D_{0}=-0.0176 /-0.0301 \\
{ }^{1} S_{0}=-0.00004 / 0.0 \\
{ }^{5} D_{0}=-0.0433 /-0.0062 \\
{ }^{1} S_{0}=0.0706 /-0.0168 \\
{ }^{1} S_{0}=0.00003 / 0.0 \\
{ }^{5} D_{0}=-0.0455 /\end{array}$} & \multirow[t]{11}{*}{$4690 / 4459$} \\
\hline & $D^{*} D^{*}$ & 0.4 & 0.6 & $\ldots$ & $\ldots$ & 0.3 & 2.0 & & \\
\hline & $D_{s}{ }^{*} D_{s}$ & 0.8 & 1.3 & $\ldots$ & $\ldots$ & 0.9 & 6.4 & & \\
\hline & $D_{s}{ }^{*} D_{s}{ }^{*}$ & 1.4 & 2.1 & $\cdots$ & $\cdots$ & 1.0 & 6.6 & & \\
\hline & $D D_{0}$ & 13 & 18.9 & $\cdots$ & $\cdots$ & 7.1 & 48 & & \\
\hline & $D D_{2}$ & 2.0 & 3.0 & $\ldots$ & $\cdots$ & 3.4 & 23 & & \\
\hline & $D_{1} D^{*}$ & 11 & 15.9 & $\cdots$ & $\cdots$ & 0.07 & 0.5 & & \\
\hline & & & & & & & & & \\
\hline & $D^{*} D_{1}^{\prime}$ & 26 & 41.3 & $\ldots$ & $\ldots$ & 0.4 & 2.9 & & \\
\hline & $D^{*} D_{2}$ & 11 & 16.1 & $\ldots$ & $\ldots$ & $\ldots$ & $\ldots$ & & \\
\hline & Total & 67 & 100 & $\ldots$ & $\ldots$ & 15 & 100 & & \\
\hline
\end{tabular}

${ }^{\mathrm{a}}$ Observed mass from the PDG [1].

TABLE III. Open-charm strong decay properties for the $2 P$ and $3 P$ charmonium states in the LP and SP models. The widths listed in the brackets are calculated with the masses of observations.

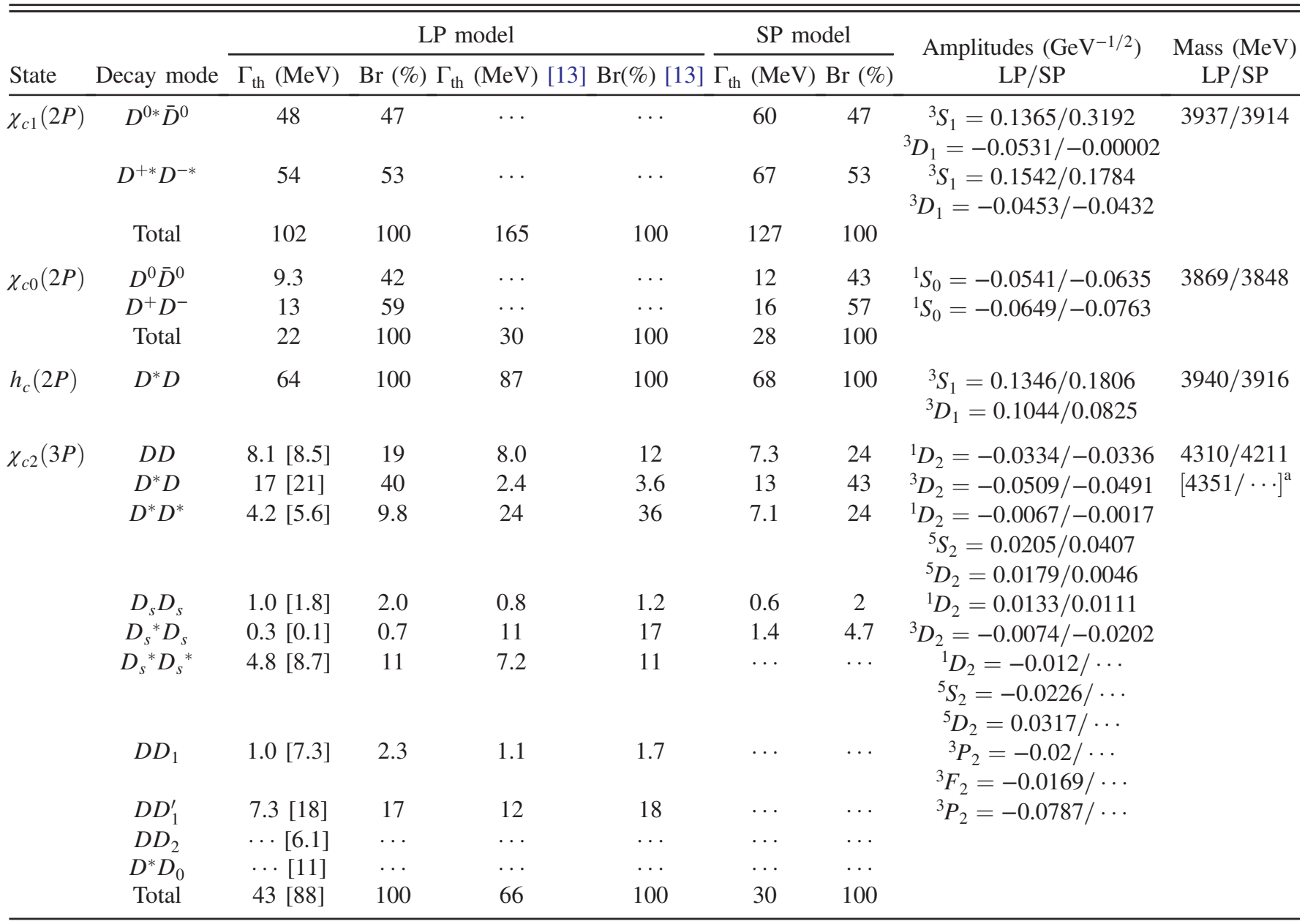


TABLE III. (Continued)

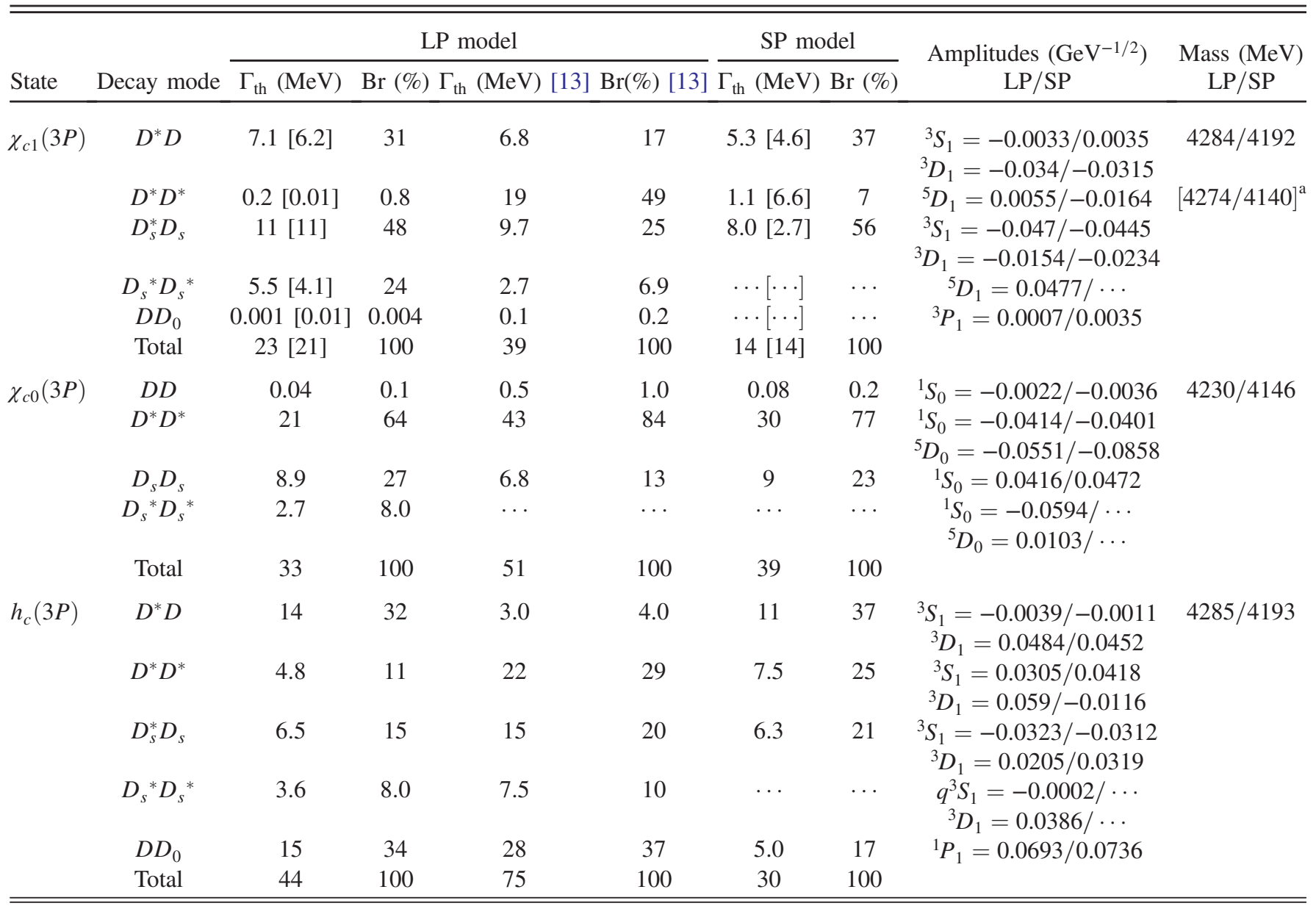

${ }^{\mathrm{a}}$ Observed mass (MeV) from the PDG [1].

which is consistent with the world average value $1.26 \pm 0.021$ from the PDG [1].

\section{2. $\psi(4040)$}

The $\psi(4040)$ resonance is assigned to be the $3{ }^{3} S_{1}$ charmonium state in the potential quark model. Four open-charm decay modes $D \bar{D}, D \bar{D}^{*}+$ c.c., $D^{*} \bar{D}^{*}$ and $D_{s} \bar{D}_{s}$ have been seen in experiment [1]. For convenience, we apply as follows the abbreviations $D D, D D^{*}, D^{*} D^{*}$ and $D_{s} D_{s}$ etc., for the corresponding charmed and anticharmed meson pairs in the final state. Its OZI allowed two-body strong decays in the LP and SP models are calculated and listed in Table I.

We find that the total width $\Gamma \sim 60 \mathrm{MeV}$ obtained in this work is slightly smaller than the world average value $80 \pm 10 \mathrm{MeV}$. The $\psi(4040)$ mainly decays into the $D^{*} D^{*}$ channel. Within the LP model, the partial width ratio,

$$
\frac{\Gamma(D D)}{\Gamma\left(D D^{*}\right)} \simeq 0.20
$$

is consistent with the measured value $0.24 \pm 0.05 \pm 0.12$ from the $B A B A R$ collaboration [59]. However, our calculation of

$$
\frac{\Gamma\left(D^{*} D^{*}\right)}{\Gamma\left(D D^{*}\right)} \simeq 3.8
$$

seems to be much larger than the measured value $0.18 \pm 0.14 \pm 0.03$ from the $B A B A R$ collaboration [59].

In the SP model the partial width ratios are

$$
\Gamma(D D): \Gamma\left(D D^{*}\right): \Gamma\left(D^{*} D^{*}\right) \simeq 1: 0.7: 10
$$

which is very different from the results of the LP model and the calculations of other works $[13,37,43]$.

It should be noted that the decay channel of $\psi(4040) \rightarrow$ $D^{*} D^{*}$ is quite sensitive to the kinematics due to the limited phase space. Furthermore, the partial width ratios extracted in various models seem not to agree with the data. Interestingly, the dominance of the $D^{*} D^{*}$ decay channel is supported by the data for $e^{+} e^{-} \rightarrow D^{*} D^{*}$ from Belle [60], and the recent analyses of Ref. [61] by solving the 
TABLE IV. Open-charm strong decay widths and amplitudes for the $4 P$ charmonium states in the LP and SP models. The widths listed in the brackets are calculated with the masses of observations.

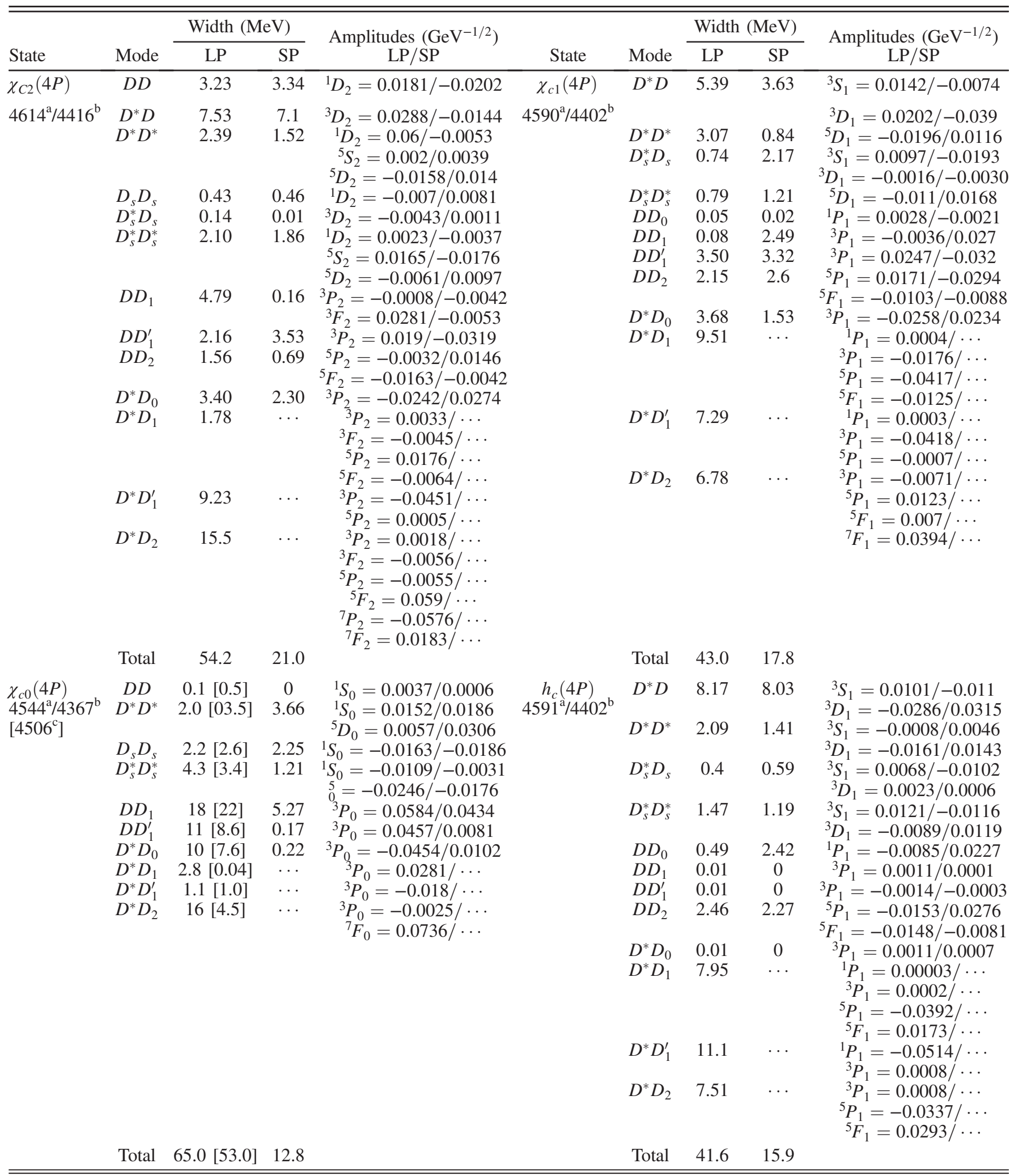

\footnotetext{
${ }^{\mathrm{a}}$ Mass $(\mathrm{MeV})$ from the LP model [17].

${ }^{\mathrm{b}}$ Mass $(\mathrm{MeV})$ from the SP model [17].

${ }^{\mathrm{c}}$ Observed mass $(\mathrm{MeV})$ at $\mathrm{LHCb}[54,55]$.
} 
TABLE V. Open-charm strong decay widths and amplitudes for the $5 P$ charmonium states in the LP and SP models. The widths listed in the brackets are calculated with the masses of observations.

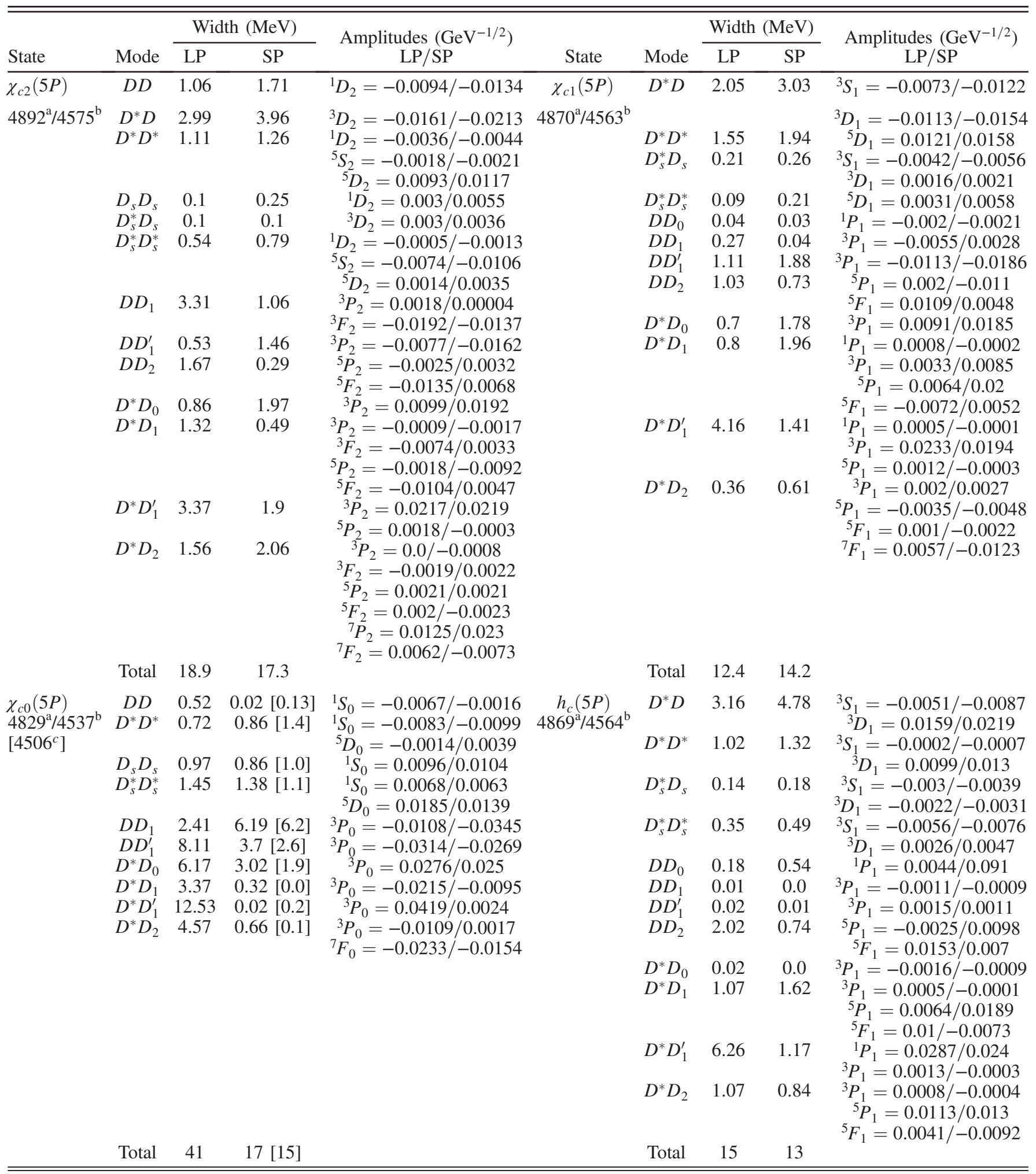

\footnotetext{
${ }^{\mathrm{a}}$ Mass $(\mathrm{MeV})$ from the LP model [17].

${ }^{\mathrm{b}}$ Mass (MeV) from the SP model [17].
} 
TABLE VI. Open-charm strong decay widths and amplitudes for the $6 P$ charmonium states in the SP models. The widths listed in the brackets are calculated with the masses of observations.

\begin{tabular}{|c|c|c|c|c|c|c|c|}
\hline State & Mode & Width (MeV) & Amplitudes $\left(\mathrm{GeV}^{-1 / 2}\right)$ & State & Mode & Width (MeV) & Amplitudes $\left(\mathrm{GeV}^{-1 / 2}\right)$ \\
\hline $\begin{array}{l}\chi_{c 2}(6 P) \\
4696^{\mathrm{a}}\end{array}$ & $\begin{array}{c}D_{s} D_{s} \\
D_{s}^{*} D_{s} \\
D_{s}^{*} D_{s}^{*} \\
\\
D D_{1}\end{array}$ & $\begin{array}{l}0.91 \\
2.26 \\
0.89 \\
\\
\\
0.13 \\
0.10 \\
0.31 \\
\\
\\
\\
1.10 \\
\\
0.56 \\
0.40 \\
\\
0.88 \\
0.16 \\
\\
\end{array}$ & $\begin{array}{c}{ }^{1} D_{2}=-0.0219 \\
{ }^{3} D_{2}=-0.0398 \\
{ }^{1} D_{2}=-0.012 \\
{ }^{5} S_{2}=-0.0284 \\
{ }^{5} D_{2}=0.0317 \\
{ }^{1} D_{2}=0.0139 \\
{ }^{3} D_{2}=0.0192 \\
{ }^{1} D_{2}=0.0024 \\
{ }^{5} S_{2}=-0.0046 \\
{ }^{5} D_{2}=-0.0064 \\
{ }^{3} P_{2}=0.006 \\
{ }^{3} F_{2}=-0.0387 \\
{ }^{3} P_{2}=0.00057 \\
{ }^{5} P_{2}=-0.0139 \\
{ }^{5} F_{2}=0.0298 \\
{ }^{3} P_{2}=0.0061 \\
{ }^{3} P_{2}=-0.0009 \\
{ }^{3} F_{2}=-0.0173 \\
{ }^{5} P_{2}=0.0001 \\
{ }^{5} F_{2}=-0.0244 \\
{ }^{3} P_{2}=0.0305 \\
{ }^{5} P_{2}=0.0032 \\
{ }^{3} P_{2}=0.00003 \\
{ }^{3} F_{2}=-0.0051 \\
{ }^{5} P_{2}=0.0031 \\
{ }^{5} F_{2}=0.0054 \\
{ }^{7} P_{2}=0.0177 \\
{ }^{7} F_{2}=0.0166 \\
{ }^{5}\end{array}$ & $\begin{array}{c}\chi_{c 1}(6 P) \\
4687^{\mathrm{a}}\end{array}$ & $\begin{array}{c}D_{s}^{*} D_{s}^{*} \\
D D_{0} \\
D D_{1} \\
D D_{1}^{\prime} \\
D D_{2} \\
\\
D^{*} D_{0} \\
D^{*} D_{1}\end{array}$ & $\begin{array}{l}1.16 \\
\\
0.73 \\
0.27 \\
\\
0.13 \\
0.03 \\
0.01 \\
1.87 \\
0.38 \\
\\
\\
1.61 \\
1.39\end{array}$ & $\begin{array}{c}{ }^{3} S_{1}=-0.0337 \\
{ }^{3} D_{1}=-0.0322 \\
{ }^{5} D_{1}=0.0447 \\
{ }^{3} S_{1}=0.0022 \\
{ }^{3} D_{1}=0.0122 \\
{ }^{5} D_{1}=0.0035 \\
{ }^{1} P_{1}=-0.0046 \\
{ }^{3} P_{1}=-0.02 \\
{ }^{3} P_{1}=-0.0188 \\
{ }^{5} P_{1}=0.0135 \\
{ }^{5} F_{1}=0.026 \\
{ }^{3} P_{1}=0.0156 \\
{ }^{1} P_{1}=0.0015 \\
{ }^{3} P_{1}=0.0064 \\
{ }^{5} P_{1}=0.0122 \\
{ }^{5} F_{1}=-0.0168 \\
{ }^{1} P_{1}=0.0010 \\
{ }^{3} P_{1}=0.0449 \\
{ }^{5} P_{1}=0.0022 \\
{ }^{3} P_{1}=0.0043 \\
{ }^{5} P_{1}=-0.0075 \\
{ }^{5} F_{1}=0.0028 \\
{ }^{7} F_{1}=0.0159\end{array}$ \\
\hline $\begin{array}{l}\chi_{c 0}(6 P) \\
4669^{\mathrm{a}} \\
{\left[4704^{\mathrm{b}}\right]}\end{array}$ & $\begin{array}{c}D D \\
D^{*} D^{*} \\
\\
D_{s} D_{s} \\
D_{s}^{*} D_{s}^{*} \\
\\
D D_{1} \\
D D_{1}^{\prime} \\
D^{*} D_{0} \\
D^{*} D_{1} \\
D^{*} D_{1}^{\prime} \\
D^{*} D_{2}\end{array}$ & $\begin{array}{c}0.05[0.004] \\
0.35[0.33] \\
\\
0.42[0.33] \\
0.73[0.72] \\
\\
2.04[1.2] \\
3.19[3.6] \\
2.59[2.7] \\
1.27[1.5] \\
2.1[3.5] \\
1.77[1.8]\end{array}$ & $\begin{array}{c}{ }^{1} S_{0}=0.0187 \\
{ }^{1} S_{0}=-0.022 \\
{ }^{5} D_{0}=0.0328 \\
{ }^{1} S_{0}=0.0028 \\
{ }^{1} S_{0}=0.0111 \\
{ }^{5} D_{0}=0.0119 \\
{ }^{3} P_{0}=-0.0005 \\
{ }^{3} P_{0}=-0.0417 \\
{ }^{3} P_{0}=0.0346 \\
{ }^{3} P_{0}=-0.0314 \\
{ }^{3} P_{0}=0.0552 \\
{ }^{3} P_{0}=-0.0156 \\
{ }^{7} F_{0}=-0.0123\end{array}$ & $\begin{array}{c}h_{c}(6 P) \\
4688^{\mathrm{a}}\end{array}$ & $\begin{array}{c}D^{*} D \\
D^{*} D^{*} \\
D_{s}^{*} D_{s} \\
D_{s}^{*} D_{s}^{*} \\
\\
D D_{0} \\
D D_{1} \\
D D_{1}^{\prime} \\
D D_{2} \\
\\
D^{*} D_{0} \\
D^{*} D_{1}\end{array}$ & $\begin{array}{l}2.28 \\
0.62 \\
0.13 \\
0.31 \\
\\
0.85 \\
0.01 \\
0.01 \\
0.45 \\
0.01 \\
0.96 \\
\\
4.09 \\
1.13\end{array}$ & $\begin{array}{c}{ }^{3} S_{1}=-0.0243 \\
{ }^{3} D_{1}=0.0451 \\
{ }^{3} S_{1}=-0.0142 \\
{ }^{3} D_{1}=0.0366 \\
{ }^{3} S_{1}=0.0021 \\
{ }^{3} D_{1}=-0.0175 \\
{ }^{3} S_{1}=-0.0077 \\
{ }^{3} D_{1}=-0.003 \\
{ }^{1} P_{1}=0.0008 \\
{ }^{3} P_{1}=-0.0027 \\
{ }^{3} P_{1}=0.0037 \\
{ }^{5} P_{1}=-0.0155 \\
{ }^{5} F_{1}=0.0371 \\
{ }^{3} P_{1}=-0.0036 \\
{ }^{1} P_{1}=-0.00003 \\
{ }^{3} P_{1}=0.0009 \\
{ }^{5} P_{1}=0.0106 \\
{ }^{5} F_{1}=0.0244 \\
{ }^{1} P_{1}=0.053 \\
{ }^{3} P_{1}=0.0024 \\
{ }^{3} P_{1}=0.0012 \\
{ }^{5} P_{1}=0.0222 \\
{ }^{5} F_{1}=0.0127\end{array}$ \\
\hline
\end{tabular}

${ }^{\mathrm{a}}$ Mass $(\mathrm{MeV})$ from the SP model [17].

${ }^{\mathrm{b}}$ Observed mass $(\mathrm{MeV})$ at LHCb $[54,55]$. 
TABLE VII. Open-charm strong decay properties for the $1 D$ and $2 D$ charmonium states in the LP and SP models.

\begin{tabular}{|c|c|c|c|c|c|c|c|c|c|}
\hline \multirow[b]{2}{*}{ State } & \multirow[b]{2}{*}{ Mode } & \multicolumn{4}{|c|}{ LP model } & \multicolumn{2}{|c|}{ SP model } & \multirow{2}{*}{$\begin{array}{c}\text { Amplitudes }\left(\mathrm{GeV}^{-1 / 2}\right) \\
\mathrm{LP} / \mathrm{SP}\end{array}$} & \multirow{2}{*}{$\begin{array}{c}\text { Mass }(\mathrm{MeV}) \\
\mathrm{LP} / \mathrm{SP}\end{array}$} \\
\hline & & $\Gamma_{\mathrm{th}}(\mathrm{MeV})$ & $\mathrm{Br}(\%)$ & $\Gamma_{\text {th }}(\mathrm{MeV})$ [13] & $\mathrm{Br}(\%)$ [13] & $\Gamma_{\mathrm{th}}(\mathrm{MeV})$ & $\mathrm{Br}(\%)$ & & \\
\hline \multirow[t]{3}{*}{$\overline{\psi_{3}(1 D)}$} & $D^{0} \bar{D}^{0}$ & 0.52 & 59 & $\cdots$ & $\ldots$ & 0.53 & 60 & \multirow{3}{*}{$\begin{array}{c}{ }^{1} F_{3}=0.015 /-0.0153 \\
{ }^{1} F_{3}=0.0127 /-0.0129\end{array}$} & \multirow[t]{3}{*}{$3811 / 3808$} \\
\hline & $D^{+} D^{-}$ & 0.36 & 41 & $\ldots$ & $\ldots$ & 0.36 & 40 & & \\
\hline & Total & 0.88 & 100 & 0.5 & 100 & 0.89 & 100 & & \\
\hline \multirow[t]{7}{*}{$\psi_{3}(2 D)$} & $D D$ & 7.2 & 11 & 24 & 16 & 5.7 & 9 & \multirow{7}{*}{$\begin{array}{c}{ }^{1} F_{3}=-0.0243 /-0.0321 \\
{ }^{3} F_{3}=-0.0756 /-0.0752 \\
{ }^{1} F_{3}=-0.0243 /-0.0191 \\
{ }^{5} P_{3}=0.0457 / 0.087 \\
{ }^{5} F_{3}=0.0534 / 0.0418 \\
{ }^{1} F_{3}=0.0348 / 0.0329 \\
{ }^{3} F_{3}=0.0316 / 0.0109\end{array}$} & \multirow[t]{7}{*}{$4172 / 4112$} \\
\hline & $D^{*} D$ & 29 & 45 & 50 & 34 & 25 & 40 & & \\
\hline & $D^{*} D^{*}$ & 20 & 31 & 67 & 45 & 28 & 44 & & \\
\hline & & & & & & & & & \\
\hline & $D_{s} D_{s}$ & 5.5 & 8.5 & 5.7 & 3.8 & 4.2 & 6.7 & & \\
\hline & $D_{s}{ }^{*} D_{s}$ & 2.9 & 4.5 & 1.2 & 0.8 & 0.2 & 0.3 & & \\
\hline & Total & 65 & 100 & 148 & 100 & 63 & 100 & & \\
\hline \multirow[t]{4}{*}{$\psi_{2}(2 D)$} & $D^{*} D$ & 28 & 38 & 34 & 37 & 22 & 44 & \multirow{4}{*}{$\begin{array}{c}{ }^{3} P_{2}=-0.0307 /-0.0238 \\
{ }^{3} F_{2}=-0.068 /-0.0661 \\
{ }^{5} P_{2}=0.0316 / 0.0491 \\
{ }^{5} F_{2}=0.0818 / 0.0633 \\
{ }^{3} P_{2}=-0.0764 /-0.0801 \\
{ }^{3} F_{2}=0.0249 / 0.0081\end{array}$} & \multirow[t]{4}{*}{$4165 / 4109$} \\
\hline & $D^{*} D^{*}$ & 28 & 38 & 32 & 35 & 18 & 36 & & \\
\hline & $D_{s}^{*} D_{s}$ & 18 & 24 & 26 & 28 & 10 & 20 & & \\
\hline & Total & 74 & 100 & 92 & 100 & 50 & 100 & & \\
\hline \multirow[t]{4}{*}{$\eta_{c 2}(2 D)$} & $D^{*} D$ & 37 & 49 & 50 & 45 & 30 & 53 & \multirow{4}{*}{$\begin{array}{c}{ }^{3} P_{2}=-0.0259 /-0.0196 \\
{ }^{3} F_{2}=0.0823 / 0.0805 \\
{ }^{3} P_{2}=0.0439 / 0.0695 \\
{ }^{3} F_{2}=0.0701 / 0.0512 \\
{ }^{3} P_{2}=-0.0618 /-0.0647 \\
{ }^{3} F_{2}=-0.03 /-0.0095\end{array}$} & \multirow[t]{4}{*}{$4164 / 4108$} \\
\hline & $D^{*} D^{*}$ & 25 & 33 & 43 & 39 & 22 & 35 & & \\
\hline & $D_{s}{ }^{*} D_{s}$ & 13 & 18 & 18 & 16 & 6.6 & 12 & & \\
\hline & Total & 75 & 100 & 111 & 100 & 59 & 100 & & \\
\hline
\end{tabular}

Lippmann-Schwinger equation and line shape studies in Ref. [62]. It shows that an improved measurement of the exclusive decays of $\psi(4040)$ and reliable extraction of its resonance parameters are needed.

\section{3. $\psi(4160)$}

The $\psi(4160)$ is assigned to be the $2^{3} D_{1}$ charmonium state in the quark model. Four open-charm decay modes $D D, D D^{*}, D^{*} D^{*}$ and $D_{s} D_{s}^{*}$ have been seen in experiments [1]. Its OZI allowed two-body strong decays using the wave functions calculated by the LP and SP models are evaluated, respectively, and the results are listed in Table I.

It shows that the measured width of $\psi(4160)$, $\Gamma \simeq 70 \pm 10 \mathrm{MeV}$, can be well described by both the LP and SP models. The decay rate of $\psi(4160)$ into $D_{s} D_{s}$ is tiny, which can explain why the $D_{s} D_{s}$ mode is not seen in experiment. In the main decay channels of $\psi(4160)$, our calculation gives

$$
\psi(4160) \rightarrow D^{*} D^{*}>D D>D D^{*},
$$

which is consistent with the result of Ref. [13]. Using the wave function calculated by the LP model, we find that

$$
\Gamma(D D): \Gamma\left(D D^{*}\right): \Gamma\left(D^{*} D^{*}\right) \simeq 4: 1: 20
$$

which is similar to the ratios, $4: 2: 20$, calculated by the SP model. However, these two ratios are very different from the measured ratios $\Gamma(D D): \Gamma\left(D D^{*}\right): \Gamma\left(D^{*} D^{*}\right) \simeq 1: 17: 50$ from the $B A B A R$ collaboration [59]. Notice that in these $P$ wave decay channels there exist obvious interfering effects between $\psi(4040)$ and $\psi(4160)$. A coherent partial wave analysis combining all these exclusive channels seems to be necessary for extracting the resonance parameters for these two states. It should be mentioned that the measured ratios cannot also be well understood in some existing models $[37,43]$.

$$
\text { 4. } \chi_{c 2}(2 P)
$$

The $X(3927)$ was observed in the $\gamma \gamma \rightarrow D \bar{D}$ process by the Belle [63] and BABAR [64] collaborations, and has been a good candidate for $\chi_{c 2}(2 P)$. We study its strong decay properties in both LP and SP models and the results are listed in Table I.

It shows that both models give similar strong decay properties for this state. The total width of $\chi_{c 2}(2 P)$ is predicted to be $\Gamma \simeq 40 \mathrm{MeV}$, which is close to the upper limit of the measurements. The $\chi_{c 2}(2 P)$ dominantly decays 
TABLE VIII. Open-charm strong decay properties for the $3 D$ charmonium states in the LP and SP models. The widths listed in the brackets are calculated with the masses of observations.

\begin{tabular}{|c|c|c|c|c|c|c|c|}
\hline \multirow[b]{2}{*}{ State } & \multirow[b]{2}{*}{ Mode } & \multicolumn{2}{|c|}{ LP model } & \multicolumn{2}{|c|}{ SP model } & \multirow{2}{*}{$\begin{array}{c}\text { Amplitudes }\left(\mathrm{GeV}^{-1 / 2}\right) \\
\text { LP/SP }\end{array}$} & \multirow{2}{*}{$\begin{array}{c}\text { Mass }(\mathrm{MeV}) \\
\mathrm{LP} / \mathrm{SP} \\
\end{array}$} \\
\hline & & $\Gamma_{\text {th }}(\mathrm{MeV})$ & $\mathrm{Br}(\%)$ & $\Gamma_{\text {th }}(\mathrm{MeV})$ & $\operatorname{Br}(\%)$ & & \\
\hline \multirow[t]{14}{*}{$\overline{\eta_{c 2}(3 D)}$} & $D^{*} D$ & 16 & 24 & 13 & 56 & \multirow{14}{*}{$\begin{array}{c}{ }^{3} P_{2}=-0.0177 /-0.0178 \\
{ }^{3} F_{2}=0.0415 / 0.0412 \\
{ }^{3} P_{2}=-0.0006 / 0.005 \\
{ }^{3} F_{2}=0.0324 / 0.0301 \\
{ }^{3} P_{2}=-0.01 /-0.0146 \\
{ }^{3} F_{2}=-0.0137 /-0.0105 \\
{ }^{3} P_{2}=-0.0198 /-0.0157 \\
{ }^{3} F_{2}=0.0051 / 0.0083 \\
{ }^{1} D_{2}=0.0048 /-0.0141 \\
{ }^{3} D_{2}=0.002 /-0.0004 \\
{ }^{3} D_{2}=-0.0024 / 0.0008 \\
{ }^{5} S_{2}=0.0397 /-0.0263 \\
{ }^{5} D_{2}=-0.0187 /-0.0144 \\
{ }^{5} G_{2}=-0.0413 /-0.0009 \\
{ }^{3} D_{2}=0.0012 /-0.0006 \\
{ }^{1} D_{2}=0.00005 / \cdots \\
{ }^{3} D_{2}=0.0013 / \cdots \\
{ }^{5} S_{2}=0.0136 / \cdots \\
{ }^{5} D_{2}=-0.0547 / \cdots \\
{ }^{5} G_{2}=-0.0141 / \cdots \\
{ }^{1} D_{2}=-0.0677 / \cdots \\
{ }^{3} D_{2}=0.0036 / \cdots \\
{ }^{5} D_{2}=-0.00003 / \cdots \\
{ }^{3} D_{2}=0.0013 / \cdots \\
{ }^{5} S_{2}=-0.0834 / \cdots \\
{ }^{5} D_{2}=-0.0208 / \cdots \\
{ }^{5} G_{2}=-0.001 / \cdots \\
\end{array}$} & \multirow[t]{14}{*}{$4478 / 4336$} \\
\hline & $D^{*} D^{*}$ & 7.3 & 11 & 5.2 & 23 & & \\
\hline & $D_{s}^{*} D_{s}$ & 1.9 & 2.8 & 1.6 & 6.9 & & \\
\hline & $D_{s}^{*} D_{s}^{*}$ & 2.2 & 3.2 & 1.1 & 4.8 & & \\
\hline & $D D_{0}$ & 0.12 & 0.2 & 0.8 & 3.5 & & \\
\hline & $D D_{1}$ & 0.02 & 0.02 & $\approx 0$ & 0 & & \\
\hline & $D D_{1}^{\prime}$ & 0.03 & 0.04 & $\approx 0$ & 0 & & \\
\hline & $D D_{2}$ & 14 & 21 & 0.9 & 3.9 & & \\
\hline & & & & & & & \\
\hline & $D^{*} D_{0}$ & 0.01 & 0.01 & $\approx 0$ & 0 & & \\
\hline & $D^{*} D_{1}$ & 7.9 & 11 & $\cdots$ & $\cdots$ & & \\
\hline & $D^{*} D_{1}^{\prime}$ & 9.6 & 14 & $\cdots$ & $\cdots$ & & \\
\hline & $D^{*} D_{2}$ & 8.1 & 12 & $\cdots$ & $\cdots$ & & \\
\hline & Total & 68 & 100 & 23 & 100 & & \\
\hline \multirow[t]{14}{*}{$\psi_{3}(3 D)$} & $D D$ & 4.5 & 6.4 & 3.4 & 14 & \multirow{14}{*}{$\begin{array}{c}{ }^{1} F_{3}=-0.0226 /-0.0212 \\
{ }^{3} F_{3}=-0.0385 /-0.0384 \\
{ }^{1} F_{3}=-0.0113 /-0.0106 \\
{ }^{5} P_{3}=-0.0083 / 0.0013 \\
{ }^{5} F_{3}=0.0248 / 0.0232 \\
{ }^{1} F_{3}=0.0145 / 0.015 \\
{ }^{3} F_{3}=0.0147 / 0.0115 \\
{ }^{1} F_{3}=-0.0009 /-0.0024 \\
{ }^{5} P_{3}=-0.0249 /-0.0224 \\
{ }^{5} F_{3}=0.002 / 0.0053 \\
{ }^{3} D_{3}=-0.0022 / 0.0075 \\
{ }^{3} G_{3}=0.0463 / 0.0134 \\
{ }^{3} D_{3}=0.0134 / 0.035 \\
{ }^{5} D_{3}=-0.0068 /-0.0119 \\
{ }^{5} G_{3}=-0.0317 /-0.0012 \\
{ }^{3} D_{3}=-0.0249 /-0.0185 \\
{ }^{3} D_{3}=0.0068 / \cdots \\
{ }^{3} G_{3}=0.0103 / \cdots \\
{ }^{5} D_{3}=0.0341 / \cdots \\
{ }^{5} G_{3}=0.0133 / \cdots \\
{ }^{3} D_{3}=-0.0573 / \cdots \\
{ }^{5} D_{3}=0.004 / \cdots \\
{ }^{3} D_{3}=0.0029 / \cdots \\
{ }^{3} G_{3}=0.0007 / \cdots \\
{ }^{5} D_{3}=-0.0053 / \cdots \\
{ }^{5} G_{3}=-0.0007 / \cdots \\
{ }^{7} S_{3}=-0.086 / \cdots \\
{ }^{7} D_{3}=-0.0418 / \cdots \\
{ }^{7} G_{3}=-0.0018 / \cdots\end{array}$} & \multirow[t]{14}{*}{$4486 / 4340$} \\
\hline & $D^{*} D$ & 12 & 17 & 9.8 & 41 & & \\
\hline & $D^{*} D^{*}$ & 5.7 & 8.1 & 3.7 & 15 & & \\
\hline & $D_{s} D_{s}$ & 1.6 & 2.2 & 1.4 & 5.8 & & \\
\hline & $D_{s}{ }^{*} D_{s}$ & 1.4 & 2.0 & 0.7 & 2.9 & & \\
\hline & $D_{s}{ }^{*} D_{s}{ }^{*}$ & 3.3 & 4.7 & 1.8 & 7.5 & & \\
\hline & $D D_{1}$ & 9.8 & 14 & 0.5 & 2.0 & & \\
\hline & $D D_{1}^{\prime}$ & 0.8 & 1.1 & 2.5 & 10 & & \\
\hline & $D D_{2}$ & 4.3 & 6.1 & 0.2 & 0.8 & & \\
\hline & $D^{*} D_{0}$ & 2.6 & 3.7 & 0.4 & 1.7 & & \\
\hline & $D^{*} D_{1}$ & 3.8 & 5.4 & $\ldots$ & $\cdots$ & & \\
\hline & $D^{*} D_{1}^{\prime}$ & 7.6 & 11 & $\cdots$ & $\cdots$ & & \\
\hline & $D^{*} D_{2}$ & 13 & 18 & $\ldots$ & $\cdots$ & & \\
\hline & Total & 70 & 100 & 24 & 100 & & \\
\hline
\end{tabular}


TABLE IX. Open-charm strong decay properties for the $3 D$ charmonium states in the LP and SP models. The widths listed in the brackets are calculated with the masses of observations.

\begin{tabular}{|c|c|c|c|c|c|c|c|}
\hline \multirow[b]{2}{*}{ State } & \multirow[b]{2}{*}{ Mode } & \multicolumn{2}{|c|}{ LP model } & \multicolumn{2}{|c|}{ SP model } & \multirow{2}{*}{$\begin{array}{c}\text { Amplitudes }\left(\mathrm{GeV}^{-1 / 2}\right) \\
\text { LP/SP }\end{array}$} & \multirow{2}{*}{$\begin{array}{c}\text { Mass }(\mathrm{MeV}) \\
\text { LP/SP }\end{array}$} \\
\hline & & $\Gamma_{\text {th }}(\mathrm{MeV})$ & $\mathrm{Br}(\%)$ & $\Gamma_{\text {th }}(\mathrm{MeV})$ & $\mathrm{Br}(\%)$ & & \\
\hline \multirow[t]{13}{*}{$\overline{\psi_{2}(3 D)}$} & $D^{*} D$ & 13 & 21 & 11 & 42 & \multirow{13}{*}{$\begin{array}{c}{ }^{3} P_{2}=-0.0208 /-0.0215 \\
{ }^{3} F_{2}=-0.0343 /-0.0339 \\
{ }^{5} P_{2}=0.0003 / 0.0038 \\
{ }^{5} F_{2}=0.0376 / 0.0351 \\
{ }^{3} P_{2}=-0.0129 /-0.0182 \\
{ }^{3} F_{2}=0.0111 / 0.0087 \\
{ }^{5} P_{2}=-0.0143 /-0.0113 \\
{ }^{5} F_{2}=0.0062 / 0.0096 \\
{ }^{1} D_{2}=0.0063 / 0.0045 \\
{ }^{3} D_{2}=-0.0011 /-0.0289 \\
{ }^{3} D_{2}=0.0239 / 0.0355 \\
{ }^{5} S_{2}=-0.0496 / 0.0294 \\
{ }^{5} D_{2}=0.0125 / 0.0091 \\
{ }^{5} G_{2}=-0.034 /-0.0008 \\
{ }^{3} D_{2}=-0.0303 /-0.0169 \\
{ }^{1} D_{2}=0.0022 / \cdots \\
{ }^{3} D_{2}=-0.029 / \cdots \\
{ }^{5} S_{2}=0.0166 / \cdots \\
{ }^{5} D_{2}=-0.0345 / \cdots \\
{ }^{5} G_{2}=0.0116 / \cdots \\
{ }^{1} D_{2}=0.0015 / \cdots \\
{ }^{3} D_{2}=-0.0557 / \cdots \\
{ }^{5} D_{2}=0.0039 / \cdots \\
{ }^{3} D_{2}=-0.0053 / \cdots \\
{ }^{5} S_{2}=0.0344 / \cdots \\
{ }^{5} D_{2}=0.0052 / \cdots \\
{ }^{5} G_{2}=-0.0003 / \cdots \\
{ }^{7} D_{2}=-0.0216 / \cdots \\
{ }^{7} G_{2}=-0.0012 / \cdots\end{array}$} & \multirow[t]{13}{*}{$4478 / 4337$} \\
\hline & $D^{*} D^{*}$ & 9.8 & 16 & 6.9 & 26 & & \\
\hline & $D_{s}^{*} D_{s}$ & 1.9 & 3.1 & 2.0 & 7.7 & & \\
\hline & $D_{s}^{*} D_{s}{ }^{*}$ & 1.3 & 2.1 & 0.7 & 2.7 & & \\
\hline & $D D_{0}$ & 0.22 & 0.4 & 0.08 & 0.3 & & \\
\hline & $D D_{1}$ & 0.01 & 0.01 & 1.9 & 7.3 & & \\
\hline & $D D_{1}^{\prime}$ & 2.5 & 4.1 & 2.5 & 9.6 & & \\
\hline & $D D_{2}$ & 15 & 24 & 1.0 & 3.8 & & \\
\hline & $D^{*} D_{0}$ & 3.7 & 6.1 & 0.3 & 1.2 & & \\
\hline & $D^{*} D_{1}$ & 5.7 & 9.3 & $\ldots$ & $\ldots$ & & \\
\hline & $D^{*} D_{1}^{\prime}$ & 6.5 & 11 & $\ldots$ & $\ldots$ & & \\
\hline & $D^{*} D_{2}$ & 1.9 & 3.1 & $\ldots$ & $\ldots$ & & \\
\hline & Total & 61 & 100 & 26 & 100 & & \\
\hline \multirow[t]{13}{*}{$\psi_{1}(3 D)$} & $D D$ & $2.1[1.2]$ & 3.3 & $3.0[3.4]$ & 15 & \multirow{13}{*}{$\begin{array}{c}{ }^{1} P_{1}=0.0156 / 0.0202 \\
{ }^{3} P_{1}=-0.0068 /-0.0089 \\
{ }^{1} P_{1}=-0.0061 /-0.0079 \\
{ }^{5} P_{1}=0.0027 / 0.0035 \\
{ }^{5} F_{1}=0.0402 / 0.0376 \\
{ }^{1} P_{1}=0.0053 / 0.0046 \\
{ }^{3} P_{2}=-0.0159 /-0.0182 \\
{ }_{1} P_{1}=0.012 / 0.007 \\
{ }^{5} P_{1}=-0.0054 /-0.0031 \\
{ }^{5} F_{1}=0.0163 / 0.0155 \\
{ }^{3} S_{1}=-0.0684 /-0.0295 \\
{ }^{3} D_{1}=0.0175 / 0.0317 \\
{ }^{3} S_{1}=-0.00004 / 0.0 \\
{ }^{3} D_{1}=0.0377 / 0.0314 \\
{ }^{5} D_{1}=0.0333 / \cdots \\
{ }^{3} D_{1}=-0.044 / \cdots \\
{ }^{3} S_{1}=0.0347 / \cdots \\
{ }^{3} D_{1}=0.0273 / \cdots \\
{ }^{5} D_{1}=-0.0437 / \cdots \\
{ }^{3} S_{1}=0.00004 / \cdots \\
{ }^{3} D_{1}=-0.0354 / \cdots \\
{ }^{5} D_{1}=0.002 / \cdots\end{array}$} & \multirow{13}{*}{$\begin{array}{c}4456 / 4324 \\
{[4415 / 4341]}\end{array}$} \\
\hline & $D^{*} D$ & $0.4[0.02]$ & 0.6 & $0.5[0.8]$ & 2.5 & & \\
\hline & $D^{*} D^{*}$ & $11[6.4]$ & 17 & $8.1[10]$ & 42 & & \\
\hline & $D_{s} D_{s}$ & $0.2[0.56]$ & 0.3 & $0.1[0.05]$ & 0.5 & & \\
\hline & $D_{s}{ }^{*} D_{s}$ & $1.6[2.1]$ & 2.5 & $1.6[1.4]$ & 8.2 & & \\
\hline & $D_{s}{ }^{*} D_{s}{ }^{*}$ & $2.2[2.9]$ & 3.4 & $0.9[1.1]$ & 4.6 & & \\
\hline & $D D_{1}$ & 21 [26] & 33 & 3.6 [9.7] & 19 & & \\
\hline & $D D_{1}^{\prime}$ & $5.8[7.1]$ & 9.1 & $1.6[3.2]$ & 8.2 & & \\
\hline & $D D_{2}$ & $4.1[6.2]$ & 6.4 & $\cdots[0.5]$ & $\ldots$ & & \\
\hline & $D^{*} D_{0}$ & $7.2[8.7]$ & 11 & $\cdots[0.7]$ & $\ldots$ & & \\
\hline & $D^{*} D_{1}$ & $6.8[0]$ & 10 & $\cdots[\cdots]$ & $\ldots$ & & \\
\hline & $D^{*} D_{1}^{\prime}$ & $1.8[0]$ & 2.8 & $\cdots[\cdots]$ & $\ldots$ & & \\
\hline & Total & 64 [61] & 100 & $20[31]$ & 100 & & \\
\hline
\end{tabular}


into the $D D$ channel, while the decay rate into the $D^{*} D$ channel is also sizable. The branching fraction $\operatorname{Br}\left[\chi_{c 2}(2 P) \rightarrow D^{*} D\right]$ can reach up to $\sim 40 \%$. In the LP model, the partial width ratio is found to be

$$
\frac{\Gamma\left(D^{*} D\right)}{\Gamma(D D)} \simeq 0.55
$$

which is slightly smaller than the ratio 0.68 obtained in the SP model. The $D^{*} D$ decay mode can be searched in $e^{+} e^{-} \rightarrow \gamma D^{*} D$ which is also the channel accessible for $X(3872)$ as predicted in Ref. [65].

\section{B. Candidates of higher $c \bar{c}$ states with $J^{P C}=1^{--}$}

The higher vector charmonium states, $\psi(4 S), \psi(5 S)$ and $\psi_{1}(3 D)$, are still not well established. During the past decade, several $J^{P C}=1^{--}$charmonium-like states, $Y(4230,4260), Y(4360)$ and $Y(4660)$, have been observed in experiment [1]. Some of them exhibit unusual properties that are very different from the expectations as conventional $c \bar{c}$ states. In addition, although $\psi(4415)$ has been well established in experiment, its structure and quark model assignment still need to be studied.

\section{1. $Y(4230,4260)$}

The $Y(4260)$ state turns out to be a mysterious state from the very beginning. It was first reported by the $B A B A R$ collaboration in the initial state radiation $e^{+} e^{-} \rightarrow$ $\gamma_{\text {ISR }} J / \psi \pi^{+} \pi^{-}$[66], and then confirmed by CLEO-c [67] and Belle [68] experiments in the same channel. However, its presence in open charm decay channels is not obvious at all, which has provoked a lot of theoretical interpretations in the literature. Comprehensive reviews can be found in several recent review articles [3,4,6-8]. Recently, following the discovery of charged charmonium-like state $Z_{c}(3900)$ in $e^{+} e^{-} \rightarrow J / \psi \pi \pi$ at the c.m. energy of $4.26 \mathrm{GeV}$ [69], the BESIII collaboration observed more detailed structures around the $Y(4260)$ in several exclusive decay channels, namely, $J / \psi \pi \pi$ [70], $h_{c} \pi \pi$ [71], $\omega \chi_{c 0}$ [72,73], and $D^{0} D^{*-} \pi^{+}+$c.c. [74]. In particular, in Ref. [74] by treating these two structures around 4.23 and $4.29 \mathrm{GeV}$ as from two Breit-Wigner states, a narrow resonance $Y(4230)$ and a relatively broad resonance $Y(4260)$ are extracted.

However, as studied in a series of works in Refs. [62,7579], the energy region of $Y(4260)$ is close to the first narrow $S$-wave open charm threshold $D D_{1}(2420)$. Therefore, a strong near-threshold $S$-wave coupling can dynamically generate a molecular state, which can then mix with the nearby vector charmonium state and cause nontrivial near-threshold structures. In such a hadronic molecule scenario, the structures observed in these exclusive decay channels can be accounted for by the dynamics introduced by the $D D_{1}(2420)$ threshold.

The interesting phenomena arising from the 4.26 energy region is also a challenge for potential quark models in the study of the $c \bar{c}$ spectrum. In the LP model, it is almost impossible to accommodate the mass of $Y(4230,4260)$ in the spectrum. In contrast, in the SP model the mass of $\psi(4 S)$ is found to be around $4.28 \mathrm{GeV}$. This might indicate the important role played by the $S$-wave threshold of $D D_{1}(2420)$ which can be partially accounted for by the screening effects. Therefore, if we assume that $Y(4260)$ is dominated by the $\psi(4 S)$ component in the wave function, we can investigate its decay properties as a charmonium state. As shown by the results listed in Table II, we find that $Y(4260)$ should be a very narrow state with a width of $\sim 14 \mathrm{MeV}$, and its strong decays are dominated by the $D^{*} D^{*}$ mode. Although such a result is consistent with that from Ref. [80] and several consequent works [81-83] with the assignment of $Y(4260)$ as the $\psi(4 S)$ state, the narrow width does not agree with the measured value of about $55 \pm 19 \mathrm{MeV}$ [1]. Note that such a solution also cannot be accommodated by the two-state fitting performed by Ref. [74]. Another issue is that if we assign $Y(4260)$ to $\psi(4 S)$, we will be unable to understand the decay properties of $\psi(4415)$ in the SP model at all, which will be discussed later.

\section{2. $\psi(4415)$}

In the LP model the calculated masses of $\psi(4 S)$ and $\psi_{1}(3 D)$ are located around $\sim 4.4 \mathrm{GeV}$. Thus, the $\psi(4415)$ might be a good candidate for $\psi(4 S)$ or $\psi_{1}(3 D)$ as broadly discussed in the literature. In contrast, in the SP model, the $\psi(4415)$ is suggested to be a candidate of $\psi(5 S)$. We discuss these possibilities below with details.

Assuming $\psi(4415)$ as the $\psi(4 S)$ state in the LP model, the calculated decay widths are listed in Table II. It shows that the predicted total width, $\Gamma \simeq 67 \mathrm{MeV}$, is consistent with the measured value $62 \pm 20 \mathrm{MeV}$ [1]. The main decay modes are $D D_{2}, D D_{1}^{\prime}, D D_{1}$ and $D^{*} D_{0}$. The branching fractions of $\operatorname{Br}\left[\psi(4415) \rightarrow D D_{2}, D D_{1}^{\prime}, D D_{1}, D^{*} D_{0}\right]$ are $\mathcal{O}(10 \%)$. Our results for some channels are significantly different from those in [13]. This difference is mainly due to the different wave functions of initial states. For example, in the $\psi(4415) \rightarrow D^{*} D_{0}^{*}$ case, if the wave function of initial state $\psi(4415)$ in Ref. [13] is replaced by that of our LP model, we get a larger partial wave amplitude ${ }^{3} S_{1}=-0.1426 \mathrm{GeV}^{-1 / 2}$ than ${ }^{3} S_{1}=-0.00087 \mathrm{GeV}^{-1 / 2}$ obtained in Ref. [13]. This reflects the fact that the integral part $I_{M_{L_{B}}, M_{L_{C}}}^{M_{L_{A}}, m}(\mathbf{P})$ is sensitive to the node position of the wave function of initial states.

In particular, the branching fraction of $\operatorname{Br}[\psi(4415) \rightarrow$ $\left.D D_{2}\right] \sim \mathcal{O}(10 \%)$ is consistent with the observations of the Belle collaboration [84]. The decay rates into $D^{*} D^{*}, D D$, $D^{*} D$ and $D_{s}^{*} D_{s}$ are relatively small with typical branching

\footnotetext{
${ }^{1}$ In this work, $D_{1}$ and $D_{1}^{\prime}$ stand for the narrow state $D_{1}(2420)$ and broad state $D_{1}(2430)$ listed in the PDG [1], respectively, which are considered to be mixed states via ${ }^{3} P_{1^{-}}{ }^{1} P_{1}$ mixing as defined in Ref. [53], while $D_{0}$ and $D_{2}$ stand for the states $D_{0}(2400)$ and $D_{2}(2460)$ listed in the PDG [1], respectively.
} 
fractions $\mathcal{O}(1 \%)$. Our calculation result for the decay rate of $\psi(4415) \rightarrow D_{s} D_{s}$ is tiny, i.e., $\operatorname{Br}\left[\psi(4415) \rightarrow D_{s} D_{s}\right]<$ $10^{-4}$. The present data for $e^{+} e^{-} \rightarrow D_{s}^{*} D_{s}^{*}$ are still with large uncertainties [85] though some hints of enhancement around 4.415 seem to be present. Further improved measurement is strongly recommended.

In the LP model the partial width ratio between the $D D$ and $D^{*} D^{*}$ channel is

$$
\frac{\Gamma(D D)}{\Gamma\left(D^{*} D^{*}\right)} \simeq 0.45
$$

which is close to the upper limit 0.29 measured by the $B A B A R$ collaboration [59]. The ratio between $D^{*} D$ and $D^{*} D^{*}$,

$$
\frac{\Gamma\left(D^{*} D\right)}{\Gamma\left(D^{*} D^{*}\right)} \simeq 0.26
$$

is also consistent with the measured value $0.17 \pm 0.28$ within uncertainties.

Assuming $\psi(4415)$ as the $\psi_{1}(3 D)$ state in the LP model, the calculation results are listed in Table IX. It is found that the calculated total width, $\Gamma \sim 60 \mathrm{MeV}$, is compatible with the measured value of $\psi(4415)$. The decays of $\psi_{1}(3 D)$ are governed by $D D_{1}$. The branching fraction of $\operatorname{Br}[\psi(4415) \rightarrow$ $D D_{1}$ ] can reach up to $50 \%$. The decay rates into $D^{*} D^{*}$, $D^{*} D_{0}, D D_{1}^{\prime}$ and $D D_{2}$ are sizable and with the branching fractions of $\operatorname{Br}\left[\psi(4415) \rightarrow D^{*} D^{*}, D^{*} D_{0}, D D_{1}^{\prime}, D D_{2}\right]$ at $\mathcal{O}(10 \%)$. The partial width ratio,

$$
\frac{\Gamma(D D)}{\Gamma\left(D^{*} D^{*}\right)} \simeq 0.18
$$

is in the range of $0.14 \pm 15$ measured by the $B A B A R$ collaboration [59], while the partial width ratio,

$$
\frac{\Gamma\left(D^{*} D\right)}{\Gamma\left(D^{*} D^{*}\right)} \simeq 0.03
$$

is also in the range of data $0.17 \pm 0.28$ [59].

Finally, we consider the possibility of $\psi(4415)$ as the $\psi(5 S)$ state in the SP models $[15,17]$. This is based on the assignment that in the SP model the mass of $\psi(4 S)$ is found to be around $4.28 \mathrm{GeV}$ as investigated in Sec. III B 1. With this hypothesis, the strong decay properties of $\psi(4415)$ are calculated and listed in Table II. The results from such an assignment turn out to be inconsistent with the observations of $\psi(4415)$.

In brief, it shows that the present data cannot distinguish the assignments of $\psi(4415)$ as $\psi(4 S)$ or $\psi_{1}(3 D)$ in the LP model, while its assignment as the $\psi(5 S)$ in the SP model cannot be supported. It should be mentioned that at the mass of $4.4 \mathrm{GeV}$, the nearby $S$-wave open threshold may introduce coupled-channel effects of which if the interaction is strong enough, it can dynamically generate poles in a unitarized formulation and mix with the charmonium state. A recent study of the dynamic effects arising from the nearby $D_{s 0}(2317) D_{s}^{*}$ and $D_{s 1}(2460) D_{s}$ thresholds and their impact on the property of $\psi(4415)$ can be found in Ref. [86]. Taking into account that both the $\psi(4 S)$ and $\psi_{1}(3 D)$ states are likely located within this energy region, it is also possible that there may exist two highly overlapping vector charmonium states around $4.4 \mathrm{GeV}$. More accurate measurements and more observables are needed in order to understand the vector spectrum above $4 \mathrm{GeV}$ in the future.

\section{3. $Y(4360)$}

In the vector charmonium spectrum, the $Y(4360)$ resonance was first reported by the $B A B A R$ collaboration in $e^{+} e^{-} \rightarrow \psi(2 S) \pi^{+} \pi^{-}$[87]. Later, the Belle collaboration confirmed this state in the same channel [88]. The interesting feature about $Y(4360)$ is its presence in the hidden charm decay channel but seems to be absent from the open charm decays. This is very similar to $Y(4260)$ when it was first observed in $e^{+} e^{-} \rightarrow J / \psi \pi \pi$. This mysterious state has also initiated many theoretical studies with different possible solutions $[3,6,8]$ including possible open-charm effects which can mix and shift the nearby charmonium state.

In the SP model, the mass of $\psi_{1}(3 D)$ is estimated to be $4.32 \mathrm{GeV}$. Considering only the mass position, $Y(4360)$ can be a good candidate of the $\psi_{1}(3 D)$. Our calculation results are listed in Table IX. It shows that the $\psi_{1}(3 D)$ resonance should be a very narrow state with a width of $\Gamma \simeq 20 \mathrm{MeV}$, and its decays should be dominated by the $D^{*} D^{*}, D D$ and $D D_{1}$ modes. In contrast with the experimental value of the width, i.e., $\Gamma=102 \pm 9 \mathrm{MeV}$, the calculated width of $\psi_{1}(3 D)$ is too small. Moreover, it has not been observed in open-charm decay channels. Note that the SP model has partly included the open-charm effects, the mismatching of $Y(4360)$ as the $\psi_{1}(3 D)$ state with the experimental measurement has reflected some unusual properties of $Y(4360)$.

\section{4. $Y(4660)$}

The $Y(4660)$ was observed in association with $Y(4360)$ by the Belle collaboration in $e^{+} e^{-} \rightarrow \psi(2 S) \pi \pi$ [88]. Its assignment is still controversial though by filling the lower states with some of these observed enhancements, it leaves the $\psi(5 S)$ as a possible option. However, it should be pointed out that the $S$-wave open-charm threshold $D^{*} D_{2}$ is located nearby. Therefore, possible contributions from the open-channel effects or dynamically generated state cannot be ruled out [6].

In the SP model, the mass of $\psi(5 S)$ is lower than $4.6 \mathrm{GeV}$ and in Sec. III B 2 the assignment of $\psi(4415)$ as the $\psi(5 S)$ has been discussed. The results show that $\psi(4415)$ does not favor such an assignment. In the LP model, the mass of $\psi(5 S)$ is predicted to be $4711 \mathrm{MeV}$, which is about $50 \mathrm{MeV}$ larger than the mass of $Y(4660)$. As 
the nearest state we investigate its strong decay properties as the $\psi(5 S)$, and the results are listed in Table II.

It shows that the calculated width $\sim 50 \mathrm{MeV}$ is close to the measured value $\Gamma=70 \pm 11 \mathrm{MeV}$. The main opencharm decay channels include $D D_{1}^{\prime}, D^{*} D_{0}, D^{*} D_{1}, D^{*} D_{1}^{\prime}$ and $D^{*} D_{2}$ with the branching fractions at the order of $10 \%-$ $20 \%$. As mentioned earlier, the $Y(4660)$ was observed in $e^{+} e^{-} \rightarrow \psi(2 S) \pi \pi$ instead of open-charm decay channels. Therefore, further experimental studies confirming or denying its contributions to these open-charm decay channels should be essential for determining its nature.

\section{Candidates of higher $c \bar{c}$ states with $J^{P C}=1^{++}$}

The higher $c \bar{c}$ states with $J^{P C}=1^{++}$, such as $\chi_{c 1}(2 P)$, $\chi_{c 1}(3 P)$ and $\chi_{c 1}(4 P)$, are still not established. During the past decade, several $J^{P C}=1^{++}$charmonium-like states, $X(3872), X(4140)$ and $X(4274)$, have been observed in experiments. They might be good candidates of the missing $J^{P C}=1^{++} c \bar{c}$ states, but associated by nontrivial dynamics.

\section{1. $X(\mathbf{3 8 7 2})$}

The $X(3872)$ resonance has the same quantum numbers as $\chi_{c 1}(2 P)$ (i.e., $J^{P C}=1^{++}$) but with a much lighter mass than potential quark model predictions. Its mass is close to the $D D^{*}$ threshold and makes it an ideal candidate for the $D D^{*}$ hadronic molecule. Various scenarios have been discussed in the literature for which recent reviews can be found in Refs. [3,4,6-8]. It is now broadly accepted that the $X(3872)$ has both a long-ranged molecular wave function and a short-ranged compact $c \bar{c}$ component [35,46,89-91]. It can be viewed as the mixture of the $D \bar{D}^{*}+$ c.c. molecule and $\chi_{c 1}(2 P)$ combined by a unitarized strong $S$-wave interaction [89] (see e.g., Ref. [6] for a detailed review). According to other authors the $X(3872)$ can be interpreted as $c \bar{c}$ core plus continuum (mesonmeson) components, like $D \bar{D}, D \bar{D}^{*}$, and so on as in Refs. [35,46,90,91].

We do not expect that the LP and SP model can explain the observed properties of $X(3872)$ by treating it as the $\chi_{c 1}(2 P)$ state. But as a test of the potential model calculations we consider $X(3872)$ as the $\chi_{c 1}(2 P)$ state and calculate its strong decays into $D^{0} D^{* 0}$. It shows that a strong coupling can be extracted and the dominance of the partial widths of $X(3872) \rightarrow D^{0} D^{* 0}$ (if the input mass is higher than the threshold) is consistent with the experimental observations. We also predict the width of $X(3872)$ by using its physical mass $3871.69 \pm 0.17 \mathrm{MeV}[1]$ in the ${ }^{3} P_{0}$ model, which gives a decay width of $\Gamma<7 \mathrm{MeV}$. It turns out to be that the decay width is very sensitive to the mass of $X(3872)$. As the physical mass of $X(3872)$ is very close to the $D^{0} \bar{D}^{* 0}$ threshold, the line shape of $D^{* 0}$ should be taken into account. This effect can be included by using the quasi-two-body decay formula [46,92-95]

$$
\begin{aligned}
& \Gamma\left[D^{0}(D \pi)_{D^{* 0}}\right] \\
& =2 \int_{0}^{k_{\max }} d k k^{2} \sum_{J, L}\left|\mathcal{M}^{J L}\left(X(3872) \rightarrow D^{0} \bar{D}^{0 *}\right)\right|^{2} \\
& \quad \times \frac{\Gamma_{\bar{D}^{0 *} \rightarrow \bar{D}^{0} \pi^{0}}(k)}{\left|M_{X(3872)}-E_{\bar{D}^{0 *}}(k)-E_{D^{0}}(k)\right|^{2}+\frac{1}{4} \Gamma_{\bar{D}^{0 *}}^{2}},
\end{aligned}
$$

where $\mathcal{M}^{J L}\left(X(3872) \rightarrow D^{0} \bar{D}^{0 *}\right)$ is the ${ }^{3} P_{0}$ amplitude defined in Eq. (4), $\Gamma_{\bar{D}^{0 *} \rightarrow \bar{D}^{0} \pi^{0}}(k)$ is the energy-dependent decay width of the unstable meson $\bar{D}^{* 0}$ worked out within the chiral quark model [53], and $\Gamma_{\bar{D}^{* 0}}$ is the total decay width of $\bar{D}^{* 0}$. According to PDG, we have $\Gamma_{\bar{D}^{* 0}}=$ $\Gamma_{\bar{D}^{0 *} \rightarrow \bar{D}^{0} \pi^{0}} / 0.65$ [1]. As the unstable meson $D^{* 0}$ can be slightly off shell, its momentum $k$ can range from 0 to $k_{\max }=\frac{\sqrt{M_{X(3872)}^{2}-\left(M_{\bar{D}^{0}}+M_{\pi^{0}}+M_{\left.D^{0}\right)^{2}}\right.} \sqrt{M_{X(3872)}^{2}-\left(M_{\tilde{D}^{0}}+M_{\pi^{0}}-M_{D^{0}}\right)^{2}}}{2 M_{X(3872)}}$.

Using Eq. (19), we plot the decay width of $X(3872)$ as a function of its mass within one sigma range in Fig. 2. We can see that there is no zero decay width when $X(3872)$ under $D^{0} D^{0 *}$ threshold as $D^{0 *}$ can be off shell. When the $D^{0} D^{0 *}$ threshold opens, the decay width of $X(3872)$ increases rapidly. Finally, the decay width of $X(3872)$ is predicted to be $\Gamma[X(3872)] \simeq 2 \mathrm{MeV}$ when we take the world average mass $m_{X(3872)}=3871.69 \mathrm{MeV}$ from the PDG [1]. This result is consistent with the measured widths in experiments $[96,97]$.

We mention that the radiative transitions of $X(3872) \rightarrow$ $\psi(2 S) \gamma$ and $J / \psi \gamma$ were studied in Refs. [17,46], where the $X(3872)$ was also treated as the $\chi_{c 1}(2 P)$ state. It also shows that the radiative decay properties are consistent with the observations from the BABAR [98] and LHCb [99]. This feature was regarded as evidence for $X(3872)$ being the $\chi_{c 1}(2 P)$ state. However, as studied by Refs. [100,101] the radiative decays of $X(3872)$ are shown to be driven by the short-ranged component of the wave function. Therefore, our

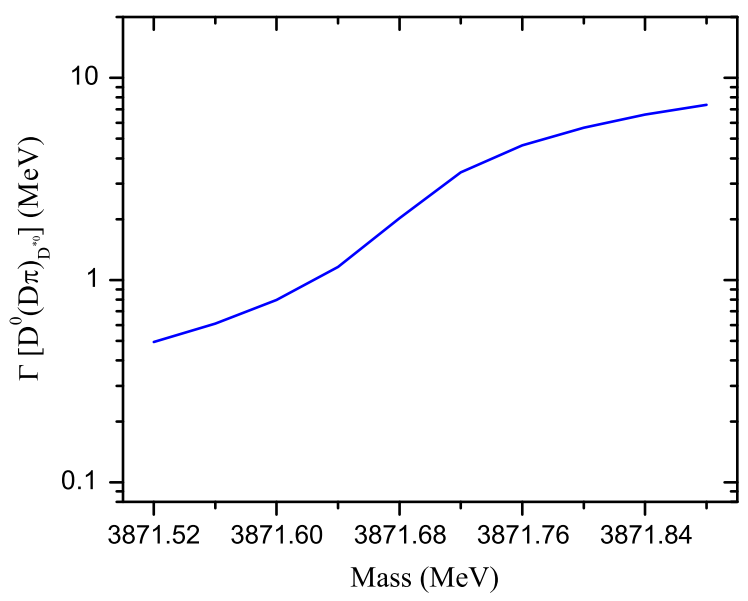

FIG. 2. The quasi-two-body decay width of $X(3872) \rightarrow$ $D^{0}(D \pi)_{D^{* 0}}$ as a function of mass by assigning $X(3872)$ as the $\chi_{c 1}(2 P)$ charmonium state. 
results actually can be regarded as a support of such a view that the short-ranged wave function is from the $\chi_{c 1}(2 P)$ state instead of concluding that it is a $\chi_{c 1}(2 P)$ state. Since the physical state of $X(3872)$ is apparently different from a conventional charmonium state, and there have been tremendous works discussing its dynamics, we do not want to overinterpret it based on our approach.

\section{2. $X(4140)$ and $X(4274)$}

In the SP model, the mass of $\chi_{c 1}(3 P)$ is predicted to be $\sim 4.19 \mathrm{MeV}$. Thus, the charmonium-like state $X(4140)$ can be a candidate for the $\chi_{c 1}(3 P)$ state. In this scenario its total width is found to be $\sim 14 \mathrm{MeV}$, which is in agreement with the world average data $19_{-7}^{+8} \mathrm{MeV}$ [1]. If $X(4140)$ corresponds to the $\chi_{c 1}(3 P)$ indeed, it may mainly decay into $D D^{*}, D^{*} D^{*}$ and $D_{s} D_{s}^{*}$ channels with comparable decay rates. The partial width ratios between these decay modes are predicted to be

$$
\frac{\Gamma\left(D D^{*}\right)}{\Gamma\left(D^{*} D^{*}\right)} \simeq 0.69, \quad \frac{\Gamma\left(D_{s} D_{s}^{*}\right)}{\Gamma\left(D^{*} D^{*}\right)} \simeq 0.40,
$$

which can be tested in future experiments.

In contrast with the LP model calculations, the mass of $\chi_{c 1}(3 P)$ is predicted to be $\sim 4.28 \mathrm{MeV}$ which is close to $X(4274)$. Assigning $X(4274)$ as the $\chi_{c 1}(3 P)$ state, we calculate its strong decays in the LP model. It shows that the LP model produces a narrow width of about $21 \mathrm{MeV}$ which is close to the lower limit of the measured width $56 \pm 11_{-11}^{+8} \mathrm{MeV}$ [54]. The ratios between different partial widths, i.e., $D D^{*}, D^{*} D^{*}$ and $D_{s} D_{s}^{*}$, are predicted to be

$$
\frac{\Gamma\left(D^{*} D^{*}\right)}{\Gamma\left(D D^{*}\right)} \simeq 2 \times 10^{-3}, \quad \frac{\Gamma\left(D_{s} D_{s}^{*}\right)}{\Gamma\left(D D^{*}\right)} \simeq 1.8 .
$$

It is interesting to note that the recent analysis of the $B^{+} \rightarrow J / \psi \phi K^{+}$process [102], and the study of the masses of $c s \overline{c s}$ tetraquark states [103] seem to favor that the $X(4274)$ may be the conventional $\chi_{c 1}(3 P)$ state. If $X(4274)$ is assigned as the $\chi_{c 1}(3 P)$ state, then one immediate question is how to understand $X(4140)$. In Ref. [102] the analysis suggests that the $X(4140)$ structure may not be a genuine resonance. There are possibilities for a nonresonance interpretation for $Y(4140)$, such as the $D_{s} D_{s}^{*}$ CUSP $[54,55]$, or $D_{s} D_{s}^{*}$ rescatterings via the open-charmed meson loops [102]. To better understand $X(4140)$ and $X(4274)$, experimental studies of their open-charm decay modes, i.e., $D D^{*}, D^{*} D^{*}$ and $D_{s} D_{s}^{*}$, are strongly recommended.

\section{Candidates of higher $c \bar{c}$ states with $J^{P C}=0^{++}$}

The higher $c \bar{c}$ states with $J^{P C}=0^{++}$, such as $\chi_{c 0}(2 P)$, $\chi_{c 0}(3 P)$ and $\chi_{c 0}(4 P)$, have not yet been established. Recently, several $J^{P C}=0^{++}$charmonium-like states,
$X^{*}(3860)$ and $X(4500,4700)$ have been observed at Belle [2] and LHCb [54], respectively. They might be good candidates for these missing $J^{P C}=0^{++} c \bar{c}$ states.

\section{1. $X^{*}(\mathbf{3 8 6 0})$}

The charmonium-like state $X^{*}(3860)$ observed in the $e^{+} e^{-} \rightarrow J / \psi D \bar{D}$ by the Belle collaboration [2] serves as a good candidate for the $\chi_{c 0}(2 P)$ state. Its measured mass and width are $3862_{-32-13}^{+26+40}$ and $201_{-67-82}^{+154+88} \mathrm{MeV}$, respectively, and fits the expectation of the $\chi_{c 0}(2 P)$ state predicted in the potential models.

Considering $X^{*}(3860)$ as the $\chi_{c 0}(2 P)$ state we study its strong decays into $D D$ in the LP and SP models. Our results are listed in Table III. Both models give a similar value of $\Gamma \simeq 22-28 \mathrm{MeV}$, which is rather narrow. Our results are consistent with that from Ref. [13]. Note that the present experimental width is not well determined. In contrast, results from different theoretical calculations are still controversial. For instance, the recent analysis of Ref. [104] found a small width about $11 \mathrm{MeV}$ for the $\chi_{c 0}(2 P)$ state [104], while the analysis of Ref. [30] in a ${ }^{3} P_{0}$ model obtained a large width of $110-180 \mathrm{MeV}$. This suggests that further precise measurement of the $X^{*}(3860)$ and more theoretical studies of the $\chi_{c 0}(2 P)$ state are necessary. We mention that tetraquark interpretations were also proposed for the nature of $X^{*}(3860)$ in the literature $[105,106]$.

\section{2. $X(4500 / 4700)$}

Recently, two $J^{P C}=0^{++}$charmonium-like states $X(4500)$ and $X(4700)$ were observed in the $J / \psi \phi$ invariant mass distributions in $B^{+} \rightarrow J / \psi \phi K^{+}$at $\mathrm{LHCb}[54,55]$. The measured widths of $X(4500)$ and $X(4700)$ are $92 \pm 21_{-20}^{+21}$ and $120 \pm 31_{-33}^{+42} \mathrm{MeV}$, respectively. To understand the nature of these two structures, different interpretations have been proposed in the literature [103,107-110]. In this work we consider the possible assignments of these two states as conventional charmonium states.

In the LP model the predicted mass of $\chi_{c 0}(4 P), M \simeq$ $4544 \mathrm{MeV}$, is close to the mass of $X(4500), 4506 \pm$ $11_{-15}^{+12} \mathrm{MeV}$. Considering the $X(4500)$ as the $\chi_{c 0}(4 P)$ state, we study its strong decay properties and the results are listed in Table IV. It is shown that the calculated total width, $\Gamma \sim 50 \mathrm{MeV}$, is compatible with the measured value of $X(4500)$ within its uncertainties. In Ref. [111], the $X(4500)$ was also considered as the conventional $\chi_{c 0}(4 P)$ state in the coupled-channel approach. If $X(4500)$ is the $\chi_{c 0}(4 P)$ state indeed, it may dominantly decay into the $D D_{1}, D D_{1}^{\prime}, D^{*} D_{0}$ and $D^{*} D_{2}$ channels, and the branching fractions are about $\mathcal{O}(10 \%)$. In contrast, the decay rate into the $D D$ channel is relatively small, which is predicted to be $\operatorname{Br}[X(4500) \rightarrow$ $D D] \sim 10^{-3}$. Such a small branching ratio may be difficult to observe in experiment. 
In the SP model the predicted mass of $\chi_{c 0}(5 P)$ is $4537 \mathrm{MeV}$, which also makes $X(4500)$ a good candidate. Assigning $X(4500)$ as the $\chi_{c 0}(5 P)$ state, the strong decay properties of $X(4500)$ are studied and the results are listed in Table V. It shows that the $\chi_{c 0}(5 P)$ state should be very narrow in the SP model with a width of $\Gamma \simeq 15 \mathrm{MeV}$. Its dominant decay modes are the $D D_{1}$ and $D D_{1}^{\prime}$ channels. One notices that the total width in the SP model is about a factor of 4 smaller than the observed width of $X(4500)$.

In the SP model the predicted mass of $\chi_{c 0}(6 P)$ is about $4669 \mathrm{MeV}$, which is very close to that of $X(4700)$. Considering $X(4700)$ as the $\chi_{c 0}(6 P)$ state, we find that the predicted width is about $16 \mathrm{MeV}$ (see Table VI), which is too narrow to be comparable with the observed width $120 \pm 31_{-33}^{+42} \mathrm{MeV}$ of $X(4700)$. Although the mass seems to fit the experimental observation, the significant discrepancy in the width raises questions on the structure of $X(4700)$. In Ref. [102], the $X(4700)$ is explained as the $\psi^{\prime} \phi$ rescattering via the $\psi^{\prime} K_{1}$ loops. The $X(4700)$ resonance might be a candidate of charmonium hybrid, for its mass is close to the charmonium hybrids with $J^{P C}=0^{++}$predicted in Ref. [10].

In brief, we find that as the $\chi_{c 0}(4 P)$ state the mass and width of $X(4500)$ can be understood in the LP model. In contrast, although the masses of both $X(4500)$ and $X(4700)$ can be described by the SP model, their widths appear to be difficult to understand.

\section{E. Candidates of higher $c \bar{c}$ states with $\boldsymbol{J}^{P C}=\mathbf{2}^{++}$}

The $X(4350)$ found by Belle [112] in the $\phi J / \psi$ mass spectrum has been a good candidate for the $\chi_{c 2}(3 P)$ state with $J^{P C}=2^{++}$. The extracted mass and width are $4350.6_{-5.1}^{+4.6} \pm 0.7$ and $13_{-9}^{+18} \pm 4 \mathrm{MeV}$, respectively. In Ref. [44] such a possible assignment was also considered.

In the LP model the calculated mass of $\chi_{c 2}(3 P)$ is about $4310 \mathrm{MeV}$, which is very close to the mass of $X(4350)$. Assigning the $X(4350)$ as the $\chi_{c 2}(3 P)$, we study its strong decays and the results are listed in Table III. Its width is found to be about $90 \mathrm{MeV}$, which is much larger than the observed value. This indeed raises questions on such an assignment. Note that the present experimental information is still very rough. Therefore, future experimental search for its decays into $D D^{*}, D D, D^{*} D^{*}$ and $D_{s}^{*} D_{s}^{*}$ are strongly recommended.

\section{F. Candidates of higher $c \bar{c}$ states with $J^{P C}=0^{-+}$}

The $X(3940)$ was first observed by the Belle collaboration in $e^{+} e^{-} \rightarrow J / \psi+X$ [113]. This state is also established in the invariant mass spectrum of $D^{*} \bar{D}$ in the $e^{+} e^{-} \rightarrow J / \psi D^{*} \bar{D}$ process [114]. The updated mass and width of $X(3940)$ are $M=3942_{-6}^{+7} \pm 6$ and $\Gamma=37_{-15}^{+26} \pm$ $8 \mathrm{MeV}$, respectively. Its decay into $D D^{*}$ but not $D D$ suggests that it has unnatural parity. The most likely interpretation of $X(3940)$ is that it is the $\eta_{c}(3 S)$ state with
$J^{P C}=0^{-+}[9]$, although the predicted mass in potential models appears to be higher than the observations.

Considering $X(3940)$ as the $\eta_{c}(3 S)$ state, we analyze its strong decays in both the LP and SP models and the results are listed in Table II. Due to the limited phase space, it shows that the $D D^{*}$ channel is the only open-charm decay for $X$ (3940). Furthermore, in the SP model the decay width is predicted to be about $40 \mathrm{MeV}$, which is in good agreement with the measurements. We also mention that the width is consistent with the calculation result from the Bethe-Salpeter method [39]. So far, the $X(3940)$ turns out to be a good candidate for $\eta_{c}(3 S)$ according to its strong decay properties.

\section{SUMMARY}

In this work we carry out a systematical study of the open-charm strong decays of the higher charmonium states up to the mass of the $6 P$ multiplet within the ${ }^{3} P_{0}$ model. The wave functions of the initial charmonium states are adopted from the calculations by the LP and SP models in our previous work. Several key results from this study can be learned here:

(i) The decay widths for the well-established charmonium states $\psi(3770), \psi(4040), \psi(4160)$ and $\chi_{c 2}(2 P)$ can be reasonably described in the LP and SP models, although for $\psi(4040)$ and $\psi(4160)$ some partial width ratios between the open-charm decay modes appear to have large discrepancies with the data.

(ii) In the LP model, the $\psi(4415)$ favors the $\psi(4 S)$ assignment, while the possibility of $\psi(4415)$ as the $\psi_{1}(3 D)$ state cannot be excluded. There may exist two highly overlapped $J^{P C}=1^{--}$charmonium states around $4.4 \mathrm{GeV}$.

(iii) In the LP model, the charmonium-like states $X(4500)$ and $X(4660)$ could be assigned as the $\chi_{c 0}(4 P)$ and $\psi(5 S)$ states, respectively.

(iv) The newly observed state $X^{*}(3860)$ seems to be too broad if it is classified as the $\chi_{c 0}(2 P)$ state. Our calculation shows that the $\chi_{c 0}(2 P)$ state should be a narrow state with $\Gamma \simeq 30 \mathrm{MeV}$.

(v) The $X(3940)$ favors the assignment as the $\eta_{c}(3 S)$ state, although the predicted mass in the potential models is somehow higher than the experimental data.

(vi) The $X(4140)$ resonance may be a good candidate of the $\chi_{c 1}(3 P)$ state in the SP model, while in the LP model $X(4274)$ seems to favor the $\chi_{c 1}(3 P)$ state. However, since it is difficult to accommodate these two states in the same model, future studies of these two states are needed for a better understanding of their nature.

(vii) The vector charmonium-like states, $Y(4230 / 4260)$ and $Y(4360)$, and the scalar charmonium state $X(4700)$ cannot be accommodated by the conventional charmonium spectrum. 
In brief, we show that a systematic study of the charmonium spectrum in the LP and SP models is useful for identifying unusual features arising from some of those higher charmonium-like states recently observed in experiment. We anticipate that future experimental measurements of some of those open-charm decay channels should be helpful for pinning down both conventional and unconventional charmonium states and provide more insights into the underlying dynamics in the charmonium mass regime.

\section{ACKNOWLEDGMENTS}

This work is supported, in part, by the National Natural Science Foundation of China under Grants No. 11775078, No. 11405053, No. 11705056, No. 11425525, No. 11521505, and No. 11261130311; by DFG and NSFC through funds provided to the Sino-German CRC 110 "Symmetries and the Emergence of Structure in QCD" (NSFC Grant No. 11261130311), and National Key Basic Research Program of China under Contract No. 2015CB856700.
[1] C. Patrignani et al. (Particle Data Group), Review of particle physics, Chin. Phys. C 40, 100001 (2016).

[2] K. Chilikin et al. (Belle Collaboration), Observation of an alternative $\chi_{c 0}(2 P)$ candidate in $e^{+} e^{-} \rightarrow J / \psi D \bar{D}$, Phys. Rev. D 95, 112003 (2017).

[3] H. X. Chen, W. Chen, X. Liu, and S. L. Zhu, The hiddencharm pentaquark and tetraquark states, Phys. Rep. 639, 1 (2016).

[4] S. L. Olsen, A new hadron spectroscopy, Front. Phys. China 10, 121 (2015).

[5] A. Esposito, A. Pilloni, and A. D. Polosa, Multiquark resonances, Phys. Rep. 668, 1 (2017).

[6] F. K. Guo, C. Hanhart, U. G. Meißner, Q. Wang, Q. Zhao, and B. S. Zou, Hadronic molecules, Rev. Mod. Phys. 90, 015004 (2018).

[7] S. L. Olsen, T. Skwarnicki, and D. Zieminska, Nonstandard heavy mesons and baryons: Experimental evidence, Rev. Mod. Phys. 90, 015003 (2018).

[8] R. F. Lebed, R. E. Mitchell, and E. S. Swanson, Heavyquark QCD exotica, Prog. Part. Nucl. Phys. 93, 143 (2017).

[9] E. Eichten, S. Godfrey, H. Mahlke, and J. L. Rosner, Quarkonia and their transitions, Rev. Mod. Phys. 80, 1161 (2008).

[10] P. Guo, A. P. Szczepaniak, G. Galata, A. Vassallo, and E. Santopinto, Heavy quarkonium hybrids from Coulomb gauge QCD, Phys. Rev. D 78, 056003 (2008).

[11] E. Eichten, K. Gottfried, T. Kinoshita, J. B. Kogut, K. D. Lane, and T. M. Yan, The Spectrum of Charmonium, Phys. Rev. Lett. 34, 369 (1975); Erratum, Phys. Rev. Lett.36, 1276(E) (1976).

[12] S. Godfrey and N. Isgur, Mesons in a relativized quark model with chromodynamics, Phys. Rev. D 32, 189 (1985).

[13] T. Barnes, S. Godfrey, and E. S. Swanson, Higher charmonia, Phys. Rev. D 72, 054026 (2005).

[14] S. Godfrey and K. Moats, Bottomonium mesons and strategies for their observation, Phys. Rev. D 92, 054034 (2015).

[15] B. Q. Li and K. T. Chao, Higher charmonia and X,Y,Z states with screened potential, Phys. Rev. D 79, 094004 (2009).
[16] B. Q. Li, C. Meng, and K. T. Chao, Search for $\chi_{c_{J}}(2 P)$ from higher charmonium E1 transitions and X,Y,Z states, arXiv:1201.4155.

[17] W. J. Deng, H. Liu, L. C. Gui, and X. H. Zhong, Charmonium spectrum and their electromagnetic transitions with higher multipole contributions, Phys. Rev. D 95, 034026 (2017).

[18] L. Micu, Decay rates of meson resonances in a quark model, Nucl. Phys. B10, 521 (1969).

[19] A. Le Yaouanc, L. Oliver, O. Pene, and J. C. Raynal, Naive quark pair creation model of strong interaction vertices, Phys. Rev. D 8, 2223 (1973).

[20] A. Le Yaouanc, L. Oliver, O. Pene, and J.-C. Raynal, Naive quark pair creation model and baryon decays, Phys. Rev. D 9, 1415 (1974).

[21] J. Ferretti and E. Santopinto, Open-flavor strong decays of open-charm and open-bottom mesons in the ${ }^{3} P_{0}$ model, Phys. Rev. D 97, 114020 (2018).

[22] A. Le Yaouanc, L. Oliver, O. Pene, and J.-C. Raynal, Strong decays of $\psi^{\prime \prime}(4.028)$ as a radial excitation of charmonium, Phys. Lett. B 71, 397 (1977).

[23] A. Le Yaouanc, L. Oliver, O. Pene, and J. C. Raynal, Why is $\psi^{\prime \prime \prime}(4.414)$ so narrow?, Phys. Lett. B 72, 57 (1977).

[24] A. Arneodo, J. L. Femenias, and F. Guerin, Strong decay of $\psi^{\prime \prime}(4.03)$ and $\psi(4.16)$ as radial excitations of charmonium, Z. Phys. C 3, 37 (1979).

[25] J. Segovia, A. M. Yasser, D. R. Entem, and F. Fernandez, $J^{P C}=1^{--}$hidden charm resonances, Phys. Rev. D 78, 114033 (2008).

[26] H. Wang, Z. Yan, and J. Ping, Radially excited states of $\eta_{c}$, Eur. Phys. J. C 75, 196 (2015).

[27] Y.C. Yang, Z. Xia, and J. Ping, Are the $X(4160)$ and $X(3915)$ charmonium states?, Phys. Rev. D 81, 094003 (2010).

[28] H. Wang, Y. Yang, and J. Ping, Strong decays of $\chi_{c J}(2 P)$ and $\chi_{c J}(3 P)$, Eur. Phys. J. A 50, 76 (2014).

[29] D. Y. Chen, Where are $\chi_{c J}(3 P)$ ?, Eur. Phys. J. C 76, 671 (2016).

[30] G. L. Yu, Z. G. Wang, and Z. Y. Li, The analysis of the charmonium-like states $X^{*}(3860), X(3872), \quad X(3915)$, $X(3930)$ and $X(3940)$ according to its strong decay behaviors, Chin. Phys. C 42, 043107 (2018). 
[31] L. J. Chen, D. D. Ye, and A. Zhang, Is $Y(4008)$ possibly a $1^{--} \psi\left(3^{3} S_{1}\right)$ state?, Eur. Phys. J. C 74, 3031 (2014).

[32] P. G. Ortega, J. Segovia, D. R. Entem, and F. Fernández, Charmonium resonances in the $3.9 \mathrm{GeV} / c^{2}$ energy region and the $X(3915) / X(3930)$ puzzle, Phys. Lett. B 778, 1 (2018).

[33] J. Segovia, D. R. Entem, and F. Fernández, Scaling of the ${ }^{3} P_{0}$ strength in heavy meson strong decays, Phys. Lett. B 715, 322 (2012).

[34] P. González, A quark model study of strong decays of X(3915), J. Phys. G 44, 075004 (2017).

[35] J. Ferretti, G. Galatá, and E. Santopinto, Interpretation of the $X(3872)$ as a charmonium state plus an extra component due to the coupling to the meson-meson continuum, Phys. Rev. C 88, 015207 (2013).

[36] E. J. Eichten, K. Lane, and C. Quigg, Charmonium levels near threshold and the narrow state $X(3872) \rightarrow \pi^{+} \pi^{-} J / \psi$, Phys. Rev. D 69, 094019 (2004).

[37] E. J. Eichten, K. Lane, and C. Quigg, New states above charm threshold, Phys. Rev. D 73, 014014 (2006); Erratum, Phys. Rev. D73, 079903(E) (2006).

[38] G. J. Ding, J. J. Zhu, and M. L. Yan, Canonical charmonium interpretation for $Y(4360)$ and $Y(4660)$, Phys. Rev. D 77, 014033 (2008).

[39] Z. H. Wang, Y. Zhang, L. Jiang, T. H. Wang, Y. Jiang, and G. L. Wang, The strong decays of X(3940) and X(4160), Eur. Phys. J. C 77, 43 (2017).

[40] Y. Jiang, G. L. Wang, T. Wang, and W. L. Ju, Why $X(3915)$ is so narrow as a $\chi_{c 0}(2 P)$ state?, Int. J. Mod. Phys. A 28, 1350145 (2013).

[41] T. Wang, G. L. Wang, H. F. Fu, and W. L. Ju, Two-body strong decay of $\mathrm{Z}(3930)$ as the $\chi_{c 2}(2 P)$ state, J. High Energy Phys. 07 (2013) 120.

[42] F. E. Close and P. R. Page, Do $\psi(4040), \psi(4160)$ signal hybrid charmonium?, Phys. Lett. B 366, 323 (1996).

[43] J. Segovia, D. R. Entem, and F. Fernandez, Strong charmonium decays in a microscopic model, Nucl. Phys. A915, 125 (2013).

[44] X. Liu, Z. G. Luo, and Z. F. Sun, $X(3915)$ and $X(4350)$ as New Members in P-Wave Charmonium Family, Phys. Rev. Lett. 104, 122001 (2010).

[45] D. Ebert, R. N. Faustov, and V. O. Galkin, Strong decays of vector mesons to pseudoscalar mesons in the relativistic quark model, Phys. Lett. B 744, 1 (2015).

[46] J. Ferretti, G. Galatà, and E. Santopinto, Quark structure of the $X(3872)$ and $\chi_{b}(3 P)$ resonances, Phys. Rev. D 90, 054010 (2014).

[47] S. K. Choi et al. (Belle Collaboration), Observation of a Narrow Charmonium-like State in Exclusive $B^{ \pm} \rightarrow$ $K^{ \pm} \pi^{+} \pi^{-} J / \psi$ Decays, Phys. Rev. Lett. 91, 262001 (2003).

[48] D. Acosta et al. (CDF Collaboration), Observation of the Narrow State $X(3872) \rightarrow J / \psi \pi^{+} \pi^{-}$in $\bar{p} p$ Collisions at $\sqrt{s}=1.96 \mathrm{TeV}$, Phys. Rev. Lett. 93, 072001 (2004).

[49] M. Jacob and G. C. Wick, On the general theory of collisions for particles with spin, Ann. Phys. (N.Y.) 7, 404 (1959); 281, 774 (2000).

[50] Z. P. Li, The kaon photoproduction of nucleons in the chiral quark model, Phys. Rev. C 52, 1648 (1995).
[51] Q. Zhao, Z. P. Li, and C. Bennhold, Vector meson photoproduction with an effective Lagrangian in the quark model, Phys. Rev. C 58, 2393 (1998).

[52] X. H. Zhong, Q. Zhao, J. He, and B. Saghai, Study of $\pi^{-} p \rightarrow \eta n$ at low energies in a chiral constituent quark model, Phys. Rev. C 76, 065205 (2007).

[53] X. H. Zhong and Q. Zhao, Strong decays of heavy-light mesons in a chiral quark model, Phys. Rev. D 78, 014029 (2008).

[54] R. Aaij et al. (LHCb Collaboration), Observation of $J / \psi \phi$ Structures Consistent with Exotic States from Amplitude Analysis of $B^{+} \rightarrow J / \psi \phi K^{+}$Decays, Phys. Rev. Lett. 118, 022003 (2017).

[55] R. Aaij et al. (LHCb Collaboration), Amplitude analysis of $B^{+} \rightarrow J / \psi \phi K^{+}$decays, Phys. Rev. D 95, 012002 (2017).

[56] Y. J. Zhang, G. Li, and Q. Zhao, Towards a Dynamical Understanding of the Non- $D \bar{D}$ Decay of $\psi(3770)$, Phys. Rev. Lett. 102, 172001 (2009).

[57] Y. J. Zhang and Q. Zhao, The line shape of $\psi(3770)$ and low-lying vector charmonium resonance parameters in $e^{+} e^{-} \rightarrow D \bar{D}$, Phys. Rev. D 81, 034011 (2010).

[58] G. Y. Chen and Q. Zhao, Study of the anomalous cross section line shape of $e^{+} e^{-} \rightarrow D \bar{D}$ at $\psi(3770)$ with an effective field theory, Phys. Lett. B 718, 1369 (2013).

[59] B. Aubert et al. (BABAR Collaboration), Exclusive initialstate-radiation production of the $D \bar{D}, D^{*} \bar{D}$, and $D^{*} \bar{D}^{*}$ systems, Phys. Rev. D 79, 092001 (2009).

[60] K. Abe et al. (Belle Collaboration), Measurement of the Near-Threshold $e^{+} e^{-} \rightarrow D^{(*) \pm} D^{(*) \mp}$ Cross Section using Initial-State Radiation, Phys. Rev. Lett. 98, 092001 (2007).

[61] M. L. Du, U. G. Meißner, and Q. Wang, $P$-wave coupled channel effects in electron-positron annihilation, Phys. Rev. D 94, 096006 (2016).

[62] S. R. Xue, H. J. Jing, F. K. Guo, and Q. Zhao, Disentangling the role of the $Y(4260)$ in $e^{+} e^{-} \rightarrow D^{*} \bar{D}^{*}$ and $D_{s}^{*} \bar{D}_{s}^{*}$ via line shape studies, Phys. Lett. B 779, 402 (2018).

[63] S. Uehara et al. (Belle Collaboration), Observation of a $\chi_{c 2}^{\prime}$ Candidate in $\gamma \gamma \rightarrow D \bar{D}$ Production at BELLE, Phys. Rev. Lett. 96, 082003 (2006).

[64] B. Aubert et al. (BABAR Collaboration), Observation of the $\chi_{c 2}(2 p)$ meson in the reaction $\gamma \gamma \rightarrow D \bar{D}$ at $B A B A R$, Phys. Rev. D 81, 092003 (2010).

[65] F. K. Guo, C. Hanhart, U. G. Meißner, Q. Wang, and Q. Zhao, Production of the $X(3872)$ in charmonia radiative decays, Phys. Lett. B 725, 127 (2013).

[66] B. Aubert et al. (BABAR Collaboration), Observation of a Broad Structure in the $\pi^{+} \pi^{-} J / \psi$ Mass Spectrum Around 4.26-GeV/c², Phys. Rev. Lett. 95, 142001 (2005).

[67] Q. He et al. (CLEO Collaboration), Confirmation of the $Y(4260)$ resonance production in ISR, Phys. Rev. D 74, 091104 (2006).

[68] C. Z. Yuan et al. (Belle Collaboration), Measurement of $e^{+} e^{-} \rightarrow \pi^{+} \pi^{-} J / \psi$ Cross-Section via Initial State Radiation at Belle, Phys. Rev. Lett. 99, 182004 (2007).

[69] M. Ablikim et al. (BESIII Collaboration), Observation of a Charged Charmonium-like Structure in $e^{+} e^{-} \rightarrow \pi^{+} \pi^{-} J / \psi$ at $\sqrt{s}=4.26 \mathrm{GeV}$, Phys. Rev. Lett. 110, 252001 (2013).

[70] M. Ablikim et al. (BESIII Collaboration), Precise Measurement of the $e^{+} e^{-} \rightarrow \pi^{+} \pi^{-} J / \psi$ Cross Section at 
Center-of-Mass Energies from 3.77 to $4.60 \mathrm{GeV}$, Phys. Rev. Lett. 118, 092001 (2017).

[71] M. Ablikim et al. (BESIII Collaboration), Evidence of Two Resonant Structures in $e^{+} e^{-} \rightarrow \pi^{+} \pi^{-} h_{c}$, Phys. Rev. Lett. 118, 092002 (2017).

[72] M. Ablikim et al. (BESIII Collaboration), Study of $e^{+} e^{-} \rightarrow \omega \chi_{c J}$ at Center-of-Mass Energies from 4.21 to $4.42 \mathrm{GeV}$, Phys. Rev. Lett. 114, 092003 (2015).

[73] M. Ablikim et al. (BESIII Collaboration), Observation of $e^{+} e^{-} \rightarrow \omega \chi_{c 1,2}$ near $\sqrt{s}=4.42$ and $4.6 \mathrm{GeV}$, Phys. Rev. D 93, 011102 (2016).

[74] X. Y. Gao, C. P. Shen, and C. Z. Yuan, Resonant parameters of the Y(4220), Phys. Rev. D 95, 092007 (2017).

[75] Q. Wang, C. Hanhart, and Q. Zhao, Decoding the Riddle of $Y(4260)$ and $Z_{c}(3900)$, Phys. Rev. Lett. 111, 132003 (2013).

[76] Q. Wang, M. Cleven, F. K. Guo, C. Hanhart, U. G. Meißner, X. G. Wu, and Q. Zhao, Y(4260): Hadronic molecule versus hadrocharmonium interpretation, Phys. Rev. D 89, 034001 (2014).

[77] X. G. Wu, C. Hanhart, Q. Wang, and Q. Zhao, Probing the nature of $Y(4260)$ in the isospin violating process $Y(4260) \rightarrow J / \psi \eta \pi^{0}$, Phys. Rev. D 89, 054038 (2014).

[78] M. Cleven, Q. Wang, F. K. Guo, C. Hanhart, U. G. Meißner, and Q. Zhao, $Y(4260)$ as the first $S$-wave open charm vector molecular state?, Phys. Rev. D 90, 074039 (2014).

[79] W. Qin, S. R. Xue, and Q. Zhao, Production of $Y(4260)$ as a hadronic molecule state of $\bar{D} D_{1}+$ c.c. in $e^{+} e^{-}$annihilations, Phys. Rev. D 94, 054035 (2016).

[80] L. P. He, D. Y. Chen, X. Liu, and T. Matsuki, Prediction of a missing higher charmonium around $4.26 \mathrm{GeV}$ in $J / \psi$ family, Eur. Phys. J. C 74, 3208 (2014).

[81] D. Y. Chen, X. Liu, and T. Matsuki, Interference effect as resonance killer of newly observed charmonium-like states $Y(4320)$ and $Y(4390)$, Eur. Phys. J. C 78, 136 (2018).

[82] D. Y. Chen, X. Liu, and T. Matsuki, Search for missing $\psi(4 S)$ in the $e^{+} e^{-} \rightarrow \pi^{+} \pi^{-} \psi(2 S)$ process, Phys. Rev. D 93, 034028 (2016).

[83] D. Y. Chen, X. Liu, and T. Matsuki, Observation of $e^{+} e^{-} \rightarrow \chi_{c 0} \omega$ and missing higher charmonium $\psi(4 S)$, Phys. Rev. D 91, 094023 (2015).

[84] G. Pakhlova et al. (Belle Collaboration), Observation of $\psi(4415) \rightarrow D D_{2}(2460)$ Decay using Initial-State Radiation, Phys. Rev. Lett. 100, 062001 (2008).

[85] G. Pakhlova et al. (Belle Collaboration), Measurement of $e^{+} e^{-} \rightarrow D_{s}^{(*)+} D_{s}^{(*)-}$ cross sections near threshold using initial-state radiation, Phys. Rev. D 83, 011101 (2011).

[86] Z. Cao and Q. Zhao, The impact of $S$-wave thresholds $D_{s 1} \bar{D}_{s}+$ c.c. and $D_{s 0} \bar{D}_{s}^{*}+$ c.c. on vector charmonium spectrum, arXiv:1711.07309.

[87] B. Aubert et al. (BABAR Collaboration), Evidence of a Broad Structure at an Invariant Mass of $4.32 \mathrm{GeV} / c^{2}$ in the Reaction $e^{+} e^{-} \rightarrow \pi^{+} \pi^{-} \psi(2 S)$ Measured at $B A B A R$, Phys. Rev. Lett. 98, 212001 (2007).

[88] X. L. Wang et al. (Belle Collaboration), Observation of Two Resonant Structures in $e^{+} e^{-} \rightarrow \pi^{+} \pi^{-} \psi(2 S)$ via Initial State Radiation at Belle, Phys. Rev. Lett. 99, 142002 (2007).
[89] G. Y. Chen, W. S. Huo, and Q. Zhao, Identifying the structure of near-threshold states from the line shape, Chin. Phys. C 39, 093101 (2015).

[90] J. Ferretti and E. Santopinto, Higher mass bottomonia, Phys. Rev. D 90, 094022 (2014).

[91] J. Ferretti and E. Santopinto, Threshold effects in $\chi_{\mathrm{c}}(2 P)$ and $\chi_{\mathrm{b}}(3 P)$ multiplets and $J / \Psi \rho, J / \Psi \omega$ hidden-flavor strong decays of the $X(3872)$, arXiv:1806.02489.

[92] R. Kokoski and N. Isgur, Meson decays by flux tube breaking, Phys. Rev. D 35, 907 (1987).

[93] S. Capstick and W. Roberts, Quasi-two-body decays of nonstrange baryons, Phys. Rev. D 49, 4570 (1994).

[94] , W. Roberts and B. Silvestre-Brac, Meson decays in a quark model, Phys. Rev. D 57, 1694 (1998).

[95] J. Segovia, A. M. Yasser, D. R. Entem, and F. Fernandez, $D_{s 1}(2536)^{+}$decays and the properties of P-wave charmed strange mesons, Phys. Rev. D 80, 054017 (2009).

[96] T. Aushev et al. (Belle Collaboration), Study of the $B \rightarrow$ $X(3872)\left(\rightarrow D^{* 0} \bar{D}^{0}\right) K$ decay, Phys. Rev. D 81, 031103 (2010).

[97] B. Aubert et al. (BABAR Collaboration), Study of resonances in exclusive $B$ decays to $\bar{D}^{(*)} D^{(*)} K$, Phys. Rev. D 77, 011102 (2008).

[98] B. Aubert et al. (BABAR Collaboration), Evidence for $X(3872) \rightarrow \psi(2 S) \gamma$ in $B^{ \pm} \rightarrow X(3872) K^{ \pm}$Decays, and a Study of $B \rightarrow c \bar{c} \gamma K$, Phys. Rev. Lett. 102, 132001 (2009).

[99] R. Aaij et al. (LHCb Collaboration), Evidence for the decay $X(3872) \rightarrow \psi(2 S) \gamma$, Nucl. Phys. B886, 665 (2014).

[100] T. Mehen and R. Springer, Radiative decays $X(3872) \rightarrow$ $\psi(2 S) \gamma$ and $\psi(4040) \rightarrow X(3872) \gamma$ in effective field theory, Phys. Rev. D 83, 094009 (2011).

[101] F. K. Guo, C. Hanhart, Y. S. Kalashnikova, U. G. Meißner, and A. V. Nefediev, What can radiative decays of the $X(3872)$ teach us about its nature?, Phys. Lett. B 742, 394 (2015).

[102] X. H. Liu, How to understand the underlying structures of $X(4140), X(4274), X(4500)$ and $X(4700)$, Phys. Lett. B 766, 117 (2017).

[103] Q. F. Lü and Y. B. Dong, $X(4140), X(4274), X(4500)$, and $X(4700)$ in the relativized quark model, Phys. Rev. D 94 , 074007 (2016).

[104] Z. Y. Zhou and Z. Xiao, Understanding X(3862), X(3872), and $X(3930)$ in a Friedrichs-model-like scheme, Phys. Rev. D 96, 054031 (2017).

[105] Z. G. Wang, Analysis of the mass and width of the $X^{*}(3860)$ with QCD sum rules, Eur. Phys. J. A 53, 192 (2017).

[106] W. Chen, H. X. Chen, X. Liu, T. G. Steele, and S. L. Zhu, Mass spectra for $q c \bar{q} \bar{c}, s c \bar{s} \bar{c}, q b \bar{q} \bar{b}, s b \bar{s} \bar{b}$ tetraquark states with $J^{P C}=0^{++}$and $2^{++}$, Phys. Rev. D 96, 114017 (2017).

[107] H. X. Chen, E. L. Cui, W. Chen, X. Liu, and S. L. Zhu, Understanding the internal structures of the $X(4140)$, $X(4274), X(4500)$ and $X(4700)$, Eur. Phys. J. C 77, 160 (2017).

[108] J. Wu, Y. R. Liu, K. Chen, X. Liu, and S. L. Zhu, X(4140), $X(4270), X(4500)$ and $X(4700)$ and their $c s \bar{c} \bar{s}$ tetraquark partners, Phys. Rev. D 94, 094031 (2016).

[109] Z. G. Wang, Reanalysis of the $X(3915), X(4500)$ and $X(4700)$ with QCD sum rules, Eur. Phys. J. A 53, 19 (2017). 
[110] Z. G. Wang, Scalar tetraquark state candidates: $X(3915)$, $X(4500)$ and $X(4700)$, Eur. Phys. J. C 77, 78 (2017).

[111] P. G. Ortega, J. Segovia, D. R. Entem, and F. Fernández, Canonical description of the new LHCb resonances, Phys. Rev. D 94, 114018 (2016).

[112] C. P. Shen et al. (Belle Collaboration), Evidence for a New Resonance and Search for the $Y(4140)$ in the $\gamma \gamma \rightarrow \phi J / \psi$ Process, Phys. Rev. Lett. 104, 112004 (2010).
[113] K. Abe et al. (Belle Collaboration), Observation of a New Charmonium State in Double Charmonium Production in $e^{+} e^{-}$Annihilation at $\sqrt{s} \sim 10.6 \mathrm{GeV}$, Phys. Rev. Lett. 98, 082001 (2007).

[114] P. Pakhlov et al. (Belle Collaboration), Production of New Charmonium-like States in $e^{+} e^{-} \rightarrow J / \psi D^{*} \bar{D}$ at $\sqrt{s} \sim 10.6 \mathrm{GeV}$, Phys. Rev. Lett. 100, 202001 (2008). 